\title{
Large eddy simulation study of fully developed wind-turbine array boundary layers
}

\author{
Marc Calaf, ${ }^{1, a)}$ Charles Meneveau, ${ }^{2, b)}$ and Johan Meyers ${ }^{3, c)}$ \\ ${ }^{1}$ Laboratory of Environmental Fluid Mechanics and Hydrology, École Polytechnique Fédérale de Lausanne, \\ 1015 Lausanne, Switzerland \\ ${ }^{2}$ Department of Mechanical Engineering, Johns Hopkins University, 3400 North Charles Street, \\ Baltimore, Maryland 21218, USA \\ ${ }^{3}$ Department of Mechanical Engineering, Katholieke Universiteit Leuven, Celestijnenlaan 300A-Bus 2421, \\ B3001 Leuven, Belgium
}

(Received 13 August 2009; accepted 15 December 2009; published online 25 January 2010)

\begin{abstract}
It is well known that when wind turbines are deployed in large arrays, their efficiency decreases due to complex interactions among themselves and with the atmospheric boundary layer (ABL). For wind farms whose length exceeds the height of the ABL by over an order of magnitude, a "fully developed" flow regime can be established. In this asymptotic regime, changes in the streamwise direction can be neglected and the relevant exchanges occur in the vertical direction. Such a fully developed wind-turbine array boundary layer (WTABL) has not been studied systematically before. A suite of large eddy simulations (LES), in which wind turbines are modeled using the classical "drag disk" concept, is performed for various wind-turbine arrangements, turbine loading factors, and surface roughness values. The results are used to quantify the vertical transport of momentum and kinetic energy across the boundary layer. It is shown that the vertical fluxes of kinetic energy are of the same order of magnitude as the power extracted by the forces modeling the wind turbines. In the fully developed WTABL, the kinetic energy extracted by the wind turbines is transported into the wind-turbine region by vertical fluxes associated with turbulence. The results are also used to develop improved models for effective roughness length scales experienced by the ABL. The effective roughness scale is often used to model wind-turbine arrays in simulations of atmospheric dynamics at larger (regional and global) scales. The results from the LES are compared to several existing models for effective roughness lengths. Based on the observed trends, a modified model is proposed, showing improvement in the predicted effective roughness length. (C) 2010 American Institute of Physics. [doi:10.1063/1.3291077]
\end{abstract}

\section{INTRODUCTION}

With the recognition that wind energy may become an important contributor to the world's overall energy portfolio, both on- and off-shore wind farms are expected to become more extended and cover increasingly larger surface areas. With the characteristic height of the atmospheric boundary layer (ABL) of about $1 \mathrm{~km}$, wind farms with horizontal extents exceeding 10-20 km may therefore approach the asymptotic limit of "infinite" wind farms, and the boundary layer flow may approach the fully developed regime. While for a lone-standing wind turbine, the power extraction is due to the difference in the upstream and downstream kinetic energy fluxes, for a turbine in a fully developed wind-turbine array boundary layer (WTABL), the kinetic energy must be entrained from the flow above. Therefore, the vertical structure and turbulence physics becomes a crucial ingredient in determining the efficiency of a turbine in very large wind farms. The vertical structure can be compactly represented using horizontally averaged flow variables. The present

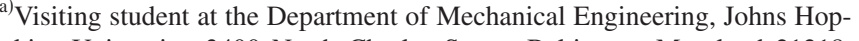
kins University, 3400 North Charles Street, Baltimore, Maryland 21218, USA. Electronic mail: marc.calaf@epfl.ch.

${ }^{b)}$ Electronic mail: meneveau@jhu.edu.

${ }^{c)}$ Electronic mail: johan.meyers@mech.kuleuven.be.
}

paper is devoted to a study of such variables based on a suite of large eddy simulations (LES) in which various parameters characterizing the wind farm are varied.

Blade aerodynamics and single turbine optimization for maximum power extraction is a well-explored topic (see, e.g., Refs. 1 and 2), building in large part on much accumulated experience from propeller aerodynamics. There is also a significant number of studies that have focused on the structure of wakes from individual wind turbines (see, e.g., Refs. 3-12). Also, a number of studies and models have been developed, dealing with superposition of wakes stemming from a finite number of wind turbines, ${ }^{13-19}$ even considering the limit of infinitely many wind turbines, as done in pioneering works by Lissaman ${ }^{20}$ (1979) and Frandsen ${ }^{19}$ (1992). Approaching the problem from the side of large atmospheric dynamics scales, wind-turbine arrays are often modeled as surface roughness elements or net drag coefficients, leading to an increased roughness length that needs to be parametrized. Examples of such studies include papers that aim at predicting interactions among wind-turbine farms with the global climate, ${ }^{21}$ regional meteorology, ${ }^{22}$ or more short-time weather patterns. ${ }^{23}$ In such simulations, the horizontal computational resolution near the ground is most often significantly coarser than the height of the boundary layer. In 
parametrizing fluxes at the ground and neglecting horizontal variations inside the smallest near-ground computational mesh, the assumption is made implicitly that the flow is "fully developed."

At present, there is relatively little information available in literature pertaining to the detailed dynamics of a fully developed boundary layer containing obstacles representing the net effects of an array of wind turbines. The current work focuses on describing the horizontally averaged structure of such a wind-turbine array boundary layer (called WTABL from here on). Using LES, various simulations of the WTABL will be undertaken. The effects of turbines will be represented in the LES using a classical "drag disk" approach. Turbine parameters that will be explored are the streamwise and spanwise turbine spacing $\left(s_{x} D\right.$ and $s_{y} D$, where $D$ is the rotor diameter), the turbine thrust coefficient $C_{T}$, and the surface roughness $z_{0, \text { lo }}$ that characterizes the ground below the wind turbines. For each of the cases, we will determine the effective "wind-farm roughness" experienced by the upper ABL. For some representative cases, we will also examine the profiles of Reynolds stresses, stresses associated with mean velocity spatial variations ("dispersive stresses") and the corresponding fluxes of kinetic energy associated with the vertical structure of the flow.

In the current study, we use a pressure-gradient driven flow in a domain of a height $H$ that is representative of the ABL (e.g., $H=1000 \mathrm{~m}$ ). The use of pressure-gradient forcing enables us to ensure that the mean flow is oriented perpendicular to the wind turbines. In the true ABL, the flow is forced through a pressure gradient arising from geostrophic balance that depends on the geostrophic velocity above the ABL. If this approach was used in the simulations, the resulting flow direction at hub height of wind turbines (e.g., $z_{h}=100 \mathrm{~m}$ ) would be an outcome of the simulation rather than an imposed parameter. This would cause unnecessary complications in interpreting the simulation results.

The main goals of this study, namely, to determine effective wind-farm roughness parameters and to examine the structure of exchanges in the region close to the wind turbines, are both based on the classical view of turbulent rough-wall boundary layers with "inner" and "outer" regions. The flow in the "outer region," at heights above $0.15 H-0.2 H$, is influenced by external effects such as Coriolis forces and the geometry of the domain. The inner flow, in the lower (say) $15 \%$ of the ABL, is determined by an inner length scale $z_{0}$, whose magnitude depends on the surface geometry and flow details in the immediate vicinity of the surface. In the classical view, the relationship between $z_{0}$ and geometric features of the surface is assumed to be independent of the outer region. Thus, for instance, effective roughness lengths of a particular rough surface can be measured in round pipes and one may apply the results to flow over the same type of surface when it is placed in a plane channel or developing boundary layer. Here, we will study the relationship between effective roughness height and characteristics of wind turbines (height, rotor diameter, horizontal spacings, loading factors, etc.) and argue that the same relations will hold generally independent of the outer flow conditions. Thus, the results obtained in a pressure driven boundary layer will be assumed to be applicable when the flow is forced by a geostrophic wind, for example, as long as the "inner scales," such as effective roughness height, windturbine height, etc., fall within the boundary layer's "inner region." This (classical) working hypothesis is limited by the fact that wind-turbine heights are close to the limits of the inner region (e.g., with hub heights and rotor diameters of $100 \mathrm{~m}$, the tip of the rotor already reaches $15 \%$ up into a $1000 \mathrm{~m}$ high $\mathrm{ABL}$ ), and furthermore, evidence for direct interactions between inner and outer dynamics in turbulent boundary layers has been observed. ${ }^{24,25}$ Nevertheless, we believe the working hypothesis can be considered a good first approximation for practical applications, especially considering the fact that the effective roughness heights that will be determined in the present work will indeed be several orders of magnitudes smaller than $H$. For one of our wind-farm cases, we tested this hypothesis by including a simulation with a larger outer scale and found that profiles match very well in the inner layer and overlap region up to five times the hub height.

In Sec. II, concepts related to the horizontally averaged WTABL are introduced, and theoretical models for the effective roughness are reviewed. The LES technique used is described in Sec. III, while the cases considered and parameters chosen for the simulation are summarized in Sec. III C. The results are presented in Sec. IV, where some representative instantaneous distributions are shown, together with mean velocity, Reynolds shear stress, and dispersive stress distributions. Section V presents the effective roughness heights deduced from the LES. These are compared with the models described above, in Sec. VI, where also an improved model is introduced based on the insights gained from the simulations. Conclusions are summarized in Sec. VII. The Appendix describes the relationship between the simulation results with pressure-gradient forcing and the case of flow forced by geostrophic wind.

\section{HORIZONTALLY AVERAGED WTABL AND EFFECTIVE ROUGHNESS OF WIND FARMS}

\section{A. Horizontally averaged WTABL structure}

We consider a fully developed, neutral stability, turbulent boundary layer forced in the $x$ direction by a mean pressure gradient, in a statistically steady state. The Reynoldsaveraged streamwise momentum equation then reads as

$$
\begin{aligned}
\bar{u} \frac{\partial \bar{u}}{\partial x}+\bar{v} \frac{\partial \bar{u}}{\partial y}+\bar{w} \frac{\partial \bar{u}}{\partial z}= & -\frac{1}{\rho} \frac{d p_{\infty}}{d x}-\frac{\partial}{\partial x} \overline{u^{\prime} u^{\prime}}-\frac{\partial}{\partial y} \overline{u^{\prime} v^{\prime}} \\
& -\frac{\partial}{\partial z} \overline{u^{\prime} w^{\prime}}+\overline{f_{x}},
\end{aligned}
$$

where the overbar denotes time averaging. Further, $u, v$, and $w$ are velocity components, the streamwise direction $x$, spanwise direction $y$, and vertical direction $z$, and $p_{\infty}$ is the averaged pressure. The fluctuating velocity is $u^{\prime}=u-\bar{u}$, with similar definitions for $v^{\prime}$ and $w^{\prime}$. Finally, $\overline{f_{x}}$ represents the time-averaged thrust force corresponding to the effect of the wind turbines on the horizontal momentum. It is nonzero only in regions where wind-turbine rotor disks are located. 
At high Reynolds numbers and away from the bottom surface and the wind-turbine surfaces, viscous stresses can be neglected.

The mean velocity distribution is a complex, threedimensional field including wakes, the boundary-layer shear, etc. A further simplification can be sought by performing a horizontal spatial average. We envision a horizontal average over directions $y$ (spanwise) and $x$ (streamwise). Horizontal averaging of this type is commonplace in studies of vegetation canopies (see, e.g., Refs. 26 and 27 for more details) and is denoted henceforth by brackets $\langle\cdots\rangle$. This spatial averaging and the assumption of horizontal statistical homogeneity yield $\langle\bar{w}\rangle=\langle\bar{v}\rangle=0$ and $\partial\langle\bar{u}\rangle / \partial x=0$. Then, the momentum equation reduces to

$$
\begin{aligned}
0 & =-\frac{1}{\rho} \frac{d p_{\infty}}{d x}-\frac{\partial}{\partial z}\left(\left\langle\overline{\left\langle u^{\prime \prime} w^{\prime \prime}\right.}\right\rangle\right)+\left\langle\overline{f_{x}}\right\rangle, \\
& =-\frac{1}{\rho} \frac{d p_{\infty}}{d x}-\frac{\partial}{\partial z}\left(\left\langle\overline{u^{\prime} w^{\prime}}\right\rangle+\left\langle\overline{\bar{u}^{\prime \prime} \bar{w}^{\prime \prime}}\right\rangle\right)+\left\langle\overline{f_{x}}\right\rangle .
\end{aligned}
$$

Here, the fluctuating velocities $u^{\prime \prime}=u-\langle\bar{u}\rangle$ and $w^{\prime \prime}=w-\langle\bar{w}\rangle$. Further, in this equation, the "dispersive stress" is commonly defined as $\left\langle\bar{u}^{\prime \prime} \bar{w}^{\prime \prime}\right\rangle$, arising due to correlations among the spatially nonhomogeneous mean horizontal and mean vertical velocities. $^{26}$

In order to also study the vertical transfer of kinetic energy from the upper ABL to the wind-turbine array, we consider the mechanical energy equation of the time- and horizontal-averaged flow, i.e., the transport equation for $\langle\bar{u}\rangle^{2} / 2$, obtained by the product of $\langle\bar{u}\rangle$ times the mean $x$-momentum equation (2),

$$
\begin{aligned}
& -\frac{1}{\rho}\langle\bar{u}\rangle \frac{d p_{\infty}}{d x}-\frac{\partial}{\partial z}\left(\left\langle\overline{u^{\prime} w^{\prime}}\right\rangle\langle\bar{u}\rangle+\left\langle\bar{u}^{\prime \prime} \bar{w}^{\prime \prime}\right\rangle\langle\bar{u}\rangle\right)+\left\langle\overline{u^{\prime} w^{\prime}}\right\rangle \frac{\partial\langle\bar{u}\rangle}{\partial z} \\
& \quad+\left\langle\bar{u}^{\prime \prime} \bar{w}^{\prime \prime}\right\rangle \frac{\partial\langle\bar{u}\rangle}{\partial z}-W_{T}=0 .
\end{aligned}
$$

Here, $W_{T}(z)=-\langle\bar{u}\rangle\left\langle\overline{f_{x}}\right\rangle$ (as will be remarked later, due to the order of averaging operations, this term differs slightly from the power density extracted from the flow by the wind turbines). It can be remarked that in the inlet region of a wind farm, the first advective term (i.e., the streamwise derivative of the mean-flow kinetic energy flux $\left.\frac{1}{2}\langle\bar{u}\rangle^{3}\right)$ is expected to be important. For very large wind-turbine arrays, the developing terms become negligible and vanish in the fully developed regime by definition. Consequently, these terms are left out of the above energy transport equation.

One of the objectives of this paper is to perform simulations of an array of wind turbines in a fully developed condition and evaluate the turbulent momentum and kinetic energy flux terms, $\left\langle\overline{\left\langle u^{\prime} w^{\prime}\right.}\right\rangle$ and $\langle\bar{u}\rangle\left\langle\overline{\left\langle u^{\prime} w^{\prime}\right.}\right\rangle$, respectively, and to compare them with the dispersive fluxes caused by spatial variations, i.e., terms $\left\langle\bar{u}^{\prime \prime} \bar{w}^{\prime \prime}\right\rangle$ and $\langle\bar{u}\rangle\left\langle\bar{u}^{\prime \prime} \bar{w}^{\prime \prime}\right\rangle$. Also, the vertical profiles of turbulent and "dispersive" dissipations, i.e., $-\left\langle\overline{u^{\prime} w^{\prime}}\right\rangle \partial\langle\bar{u}\rangle / \partial z$ and $-\left\langle\bar{u}^{\prime \prime} \bar{w}^{\prime \prime}\right\rangle \partial\langle\bar{u}\rangle / \partial z$, respectively, will be determined by averaging the simulation results. One goal is to compare the typical magnitudes of these terms and com- pare them to the mechanical power extracted by the wind turbines and to ascertain the relative effects of the dispersive terms compared to the turbulent ones.

\section{B. Models for effective roughness}

Pioneering studies regarding quantification of effective surface roughness $z_{0}$ in the ABL were done by Kutzbach ${ }^{28}$ and Lettau. ${ }^{29}$ The first efforts linking roughness effects to the effects of large wind farms in the planetary boundary layer were done by Templin, ${ }^{30}$ Newman, ${ }^{31}$ and Bossanyi et al. ${ }^{32}$ More rigorous treatments have been presented more recently by Frandsen ${ }^{19}$ and Frandsen et al. ${ }^{33}$ using the mean momentum equation and also linking the surface wind at hub height with the geostrophic wind speed.

\section{Lettau's formula}

In order to estimate an effective value for $z_{0}$ based strictly on visual site surveys and using geometric measurements to describe characteristic surface elements, Lettau, ${ }^{29}$ by considering a slight simplification of an earlier equation, ${ }^{28}$ proposed the following simple (geometric) expression: $z_{0, \text { Lett }}=0.5 h \mathrm{~A} / \mathrm{S}$, where $h$ is the average vertical extent, or obstacle height, $A$ is the silhouette area of the average obstacle, or area (measured in the vertical crosswind-lateral plane) "seen" by the wind in the approach toward a characteristic obstacle. $S$ is the specific area, measured in the horizontal plane. The numerical factor 0.5 corresponds to an assumed average drag coefficient of the characteristic individual obstacle. Setting $h$ equal to the turbine height $z_{h}$, using $A=\pi D^{2} / 4$ as the rotor disk area, and expressing $S$ in terms of the spacing between wind turbines in multiples of rotor diameter, $s_{x} D \times s_{y} D$, the Lettau formula reads as

$$
z_{0, \text { Lett }}=\frac{z_{h} \pi}{8 s_{x} s_{y}} .
$$

This model has been recently used for a study on the global weather impact of large wind farms. ${ }^{23}$

\section{Frandsen theory}

The idea of a wind farm induced ABL was introduced by Templin, ${ }^{30}$ Newman, ${ }^{31}$ Bossanyi et al., ${ }^{32}$ and further developed by Frandsen ${ }^{19}$ and Frandsen et al. ${ }^{33}$ It considers the vertical change in the natural $\mathrm{ABL}$ due to the increase in the surface roughness induced by clusters of large number of wind turbines. The concept of displacement height and effective surface roughness is commonly used in modeling flow through and above plant canopies. ${ }^{27}$

Based on momentum balance (i.e., Eq. (2) in which the "dispersive stresses" are neglected), Frandsen et al. ${ }^{19,33}$ derived an expression for the wind farm induced surface roughness. Furthermore, it is postulated that there are two equilibrium layers. The first one, the "high" layer is situated above the wind-turbine canopy, with a friction velocity $u_{* \text { wi }}$ (high denoted by subscript "hi"). In the case of fully developed flow of height $H$ driven by a pressure gradient, it is expected that $u_{* \text { hi }}=\sqrt{H \rho^{-1}\left(d p_{\infty} / d x\right)}$. At very high Reynolds number, it can also be expected that $u_{* \mathrm{hi}} \approx\left(-\left\langle\overline{u^{\prime} w^{\prime}}\right\rangle\right)^{1 / 2}$, for $H \gg z \gtrsim z_{h}$ if 
the wind turbines are in the boundary layer's inner region. The second "low" layer is assumed to exist below the windturbine array (low denoted by subscript "lo"), where the friction velocity is reduced due to the momentum lost to the wind turbines, and equals $u_{* \mathrm{l}_{\mathrm{o}}}=\sqrt{\tau_{w} / \rho}$, where $\tau_{w}$ is the stress at the ground. At very high Reynolds number, it can also be expected that $u_{* 1 \mathrm{l}} \approx\left(-\left\langle\overline{u^{\prime} w^{\prime}}\right\rangle\right)^{1 / 2}$ (for $z \lesssim z_{h}$ ). Useful estimates of these friction velocities can be made if one relates the force to the mean flow velocity at hub height. A simple model is to assume that the mean velocity in the two constant stress layers follows separate logarithmic equilibrium profiles. For the bottom and top boundary layers, one may thus write

$$
\begin{aligned}
& \langle\bar{u}\rangle_{\mathrm{lo}}(z)=\frac{u_{* \mathrm{lo}}}{\kappa} \ln \left(\frac{z}{z_{0, \mathrm{lo}}}\right) \quad \text { for } z_{0, \mathrm{lo}}<z<z_{h}, \\
& \langle\bar{u}\rangle_{\mathrm{hi}}(z)=\frac{u_{* \mathrm{hi}}}{\kappa} \ln \left(\frac{z}{z_{0, \mathrm{hi}}}\right) \quad \text { for } z>z_{h},
\end{aligned}
$$

where $\kappa$ is the usual von Kármán constant. Now assuming that the wind turbines are exposed to a typical velocity on the order of the mean velocity at hub height, $\langle\bar{u}\rangle\left(z_{h}\right)$, one may write the momentum balance equation (2) as ${ }^{33}$

$$
u_{* \mathrm{hi}}^{2}=u_{* \mathrm{lo}}^{2}+\frac{1}{2} C_{T}\left(\frac{\pi}{4} D^{2}\right)\left[\frac{u_{* \mathrm{hi}}}{\kappa} \ln \left(\frac{z_{h}}{z_{0, \mathrm{hi}}}\right)\right]^{2}\left(\frac{1}{s_{x} s_{y} D^{2}}\right) .
$$

Above, the horizontal spacings of wind turbines are $s_{x} D$ and $s_{y} D$ in the streamwise and spanwise directions, respectively, and $C_{T}$ is the turbine's thrust coefficient. The above equation may be simplified to

$$
u_{* \mathrm{hi}}^{2}=u_{* \mathrm{lo}}^{2}+\frac{1}{2} c_{\mathrm{ft}}\left(\frac{1}{\kappa} \ln \frac{z_{h}}{z_{0, \mathrm{hi}}}\right)^{2} u_{* \mathrm{hi}}^{2}
$$

where a friction coefficient $c_{\mathrm{ft}}$ based on the horizontal surface rather than frontal area simplifies the nomenclature and is defined according to

$$
c_{\mathrm{ft}}=\frac{\pi C_{T}}{4 s_{x} s_{y}}
$$

(note that this definition differs from earlier ones ${ }^{33}$ by a factor of 1/2). The approach of Frandsen et al. ${ }^{19,33}$ is to solve this equation for the unknown roughness parameter $z_{0, \text { hi }}$ appropriate for the layer above the wind turbine. The model still requires a relationship among $u_{* 1 \mathrm{o}}$ and $u_{* \mathrm{hi}}$. Neglecting the width of the wind-turbine wakes, the condition of continuity of the mean horizontally averaged velocity means equating the implied mean velocities at height $z_{h}$,

$$
\langle\bar{u}\rangle_{\mathrm{lo}}\left(z_{h}\right)=\langle\bar{u}\rangle_{\mathrm{hi}}\left(z_{h}\right) \Rightarrow u_{* 1 \mathrm{o}}=u_{* \mathrm{hi}} \frac{\ln \left(z_{h} / z_{0, \mathrm{hi}}\right)}{\ln \left(z_{h} / z_{0, \mathrm{lo}}\right)} .
$$

Substituting into Eq. (7) and solving for $z_{0, \text { hi }}$ yields the Frandsen formula for the effective roughness height,

$$
z_{0, \text { Fran }}=z_{h} \exp \left(-\frac{\kappa}{\sqrt{\frac{1}{2} c_{\mathrm{ft}}+\left[\frac{\kappa}{\ln \left(z_{h} / z_{0, \mathrm{lo}}\right)}\right]^{2}}}\right) .
$$

It can be seen that when $c_{\mathrm{ft}} \rightarrow 0$, the expression tends to the unperturbed bottom surface height $z_{0, \mathrm{lo}}$.

\section{LES METHODOLOGY AND CASES}

\section{A. Governing equations and LES codes}

In this work, we consider flow that is not thermally stratified, driven by an imposed pressure gradient. Therefore, the LES is based on the filtered incompressible NavierStokes (NS) equations for neutral flows and the continuity equation, i.e.,

$$
\begin{aligned}
& \partial_{i} \widetilde{u}_{i}=0, \\
& \partial_{t} \widetilde{u}_{i}+\partial_{j}\left(\widetilde{u}_{i} \widetilde{u}_{j}\right)=-\partial_{i} \widetilde{p}^{*}-\partial_{j} \tau_{i j}+f_{i}-\delta_{i 1} \partial_{1} p_{\infty} / \rho,
\end{aligned}
$$

where $\tilde{u}_{i}$ is the filtered velocity field and $\widetilde{p}^{*}$ is the filtered modified pressure equal to $\tilde{p} / \rho+\tau_{k k} / 3-p_{\infty} / \rho$. Further, $\tau_{i j}$ is the subgrid-scale stress term. Its deviatoric part $\left(\tau_{i j}-\delta_{i j} \tau_{k k} / 3\right)$ is modeled using an eddy viscosity subgridscale model, as discussed further below; the trace of this term $\left(\tau_{k k} / 3\right)$ is combined into the modified pressure, as is common practice in LES of incompressible flow.

The force $f_{i}$ is added for modeling the effects of the wind turbines in the momentum equation (see Sec. III B). Since simulations are done at very large Reynolds numbers and the bottom surface as well as the wind-turbine effects are parametrized, viscous stresses are neglected. In the real case of wind turbines in the ABL, the flow is forced by geostrophic wind and in the outer layer is affected by Coriolis accelerations, as discussed in the Appendix. The flow changes direction near the ground, and for a given geostrophic wind direction, the turning depends on the shear stresses (momentum exchanges) at the bottom surface. Since these are not known ahead of time, and we wish to have a mean wind that is perpendicular to the wind-turbine disks in the array to be simulated, in the simulations we prefer to use forcing with an imposed pressure gradient $\partial_{1} p_{\infty}$ in the $x_{1}$ direction. The results of the simulations, especially in the surface layer region, can still be interpreted in the context of geostrophic wind forcing, as summarized in the Appendix.

In the present study, we use two LES codes, which helps gauge the robustness of the results to details of numerical methods and implementation details. In one code (JHU-LES code), the skew-symmetric form of the NS equation is implemented. The numerical discretization follows the approach used by Moeng ${ }^{34}$ and Albertson and Parlange, ${ }^{35}$ which combines a pseudospectral discretization, and thus doubly periodic boundary conditions in the horizontal directions and a centered second-order finite differencing in the vertical direction. A second order accurate Adams-Bashforth scheme is used for time integration. The subgrid model used is the dynamic Smagorinsky model ${ }^{36}$ using the Lagrangian scaledependent version, as described in Ref. 37.

The second code (KULeuven code) also uses a pseudospectral discretization in the horizontal directions. In the 
vertical direction, a fourth-order energy-conservative finitedifference discretization ${ }^{38}$ is used. Time integration is performed using a classical four-stage fourth-order RungeKutta scheme. The subgrid-scale model is a standard Smagorinsky model ${ }^{39}$ with a constant coefficient $C_{s}=0.14$. Near the bottom surface, the Smagorinsky length scale $\lambda$ $\left(=C_{s} \Delta\right.$ far from the surface) is damped using the classic wall damping function of Mason and Thomson, ${ }^{40}$ i.e., $\lambda^{-n}=\left[C_{s} \Delta\right]^{-n}+\left[\kappa\left(z+z_{0,10}\right)\right]^{-n}$, where we take $n=3$.

In both codes, the nonlinear convective terms and the SGS stress are dealiazed using the $3 / 2$ rule. $^{41}$ Message passing interface is used to run the simulations in parallel mode, and the FFTW library ${ }^{42}$ is employed for Fourier transforms. The top boundary uses zero vertical velocity and zero shear stress boundary condition. At the bottom surface, we use a classic imposed wall stress boundary condition relating the wall stress to the velocity at the first grid-point using the standard log (Monin-Obukhov) similarity law ${ }^{34}$

$$
\begin{aligned}
& \tau_{w 1}=-\left(\frac{\kappa}{\ln z / z_{0, \mathrm{lo}}}\right)^{2}\left(\hat{\vec{u}}^{2}+\hat{\vec{v}}^{2}\right)^{0.5} \hat{\vec{u}}, \\
& \tau_{w 2}=-\left(\frac{\kappa}{\ln z / z_{0, \mathrm{lo}}}\right)^{2}\left(\hat{\vec{u}}^{2}+\hat{\tilde{v}}^{2}\right)^{0.5} \hat{\tilde{v}},
\end{aligned}
$$

where the hat on $\hat{\tilde{u}}$ and $\hat{\tilde{v}}$ represents a local average obtained by filtering the LES velocity field with filter width $2 \Delta$ (see Ref. 37 for more details about such filtering).

The applied pressure gradient $\partial_{1} p_{\infty} / \rho$ defines a reference, fixed, friction velocity $u_{* \mathrm{hi}}$ by means of $u_{* \mathrm{hi}}^{2}=H \partial_{1} p_{\infty} / \rho$. Velocities will be scaled with $u_{* \mathrm{hi}}$, while time scales will be scaled with $H / u_{* \mathrm{hi}}$. Henceforth, to simplify notation, the LES filtering "tilde" will be omitted from the indicated variables.

\section{B. Wind-turbine model}

The model for the wind turbines is based on an empirical model for the total thrust force experienced by the entire wind turbine. ${ }^{43,44}$ The total disk actuator force $F_{t}$ acting on the flow in the streamwise $x_{1}$ direction is modeled using the classical expression ${ }^{2}$

$$
F_{t}=-\frac{1}{2} \rho C_{T} U_{\infty}^{2} \frac{\pi}{4} D^{2},
$$

where $C_{T}$ is the thrust coefficient and $U_{\infty}$ is the "upstream" undisturbed reference velocity. However, in LES of windturbine arrays with significant interactions among wind turbines and wakes, this reference velocity is not readily known and would require arbitrary decisions about what upstream distance to use when specifying the velocity. ${ }^{45}$ This is unlike the LES of isolated turbines in Jimenez et al. ${ }^{43,44}$ which had negligible interactions and in which $U_{\infty}$ could be readily specified. For our simulations, it is more natural to use the velocity at the rotor disk $U_{d}$. Using classic actuator disk theory allows relating both velocities according to

$$
U_{\infty}=\frac{U_{d}}{(1-a)},
$$

where $a$ is the induction factor. ${ }^{2}$ Moreover, modeling the thrust forces acting on the fluid due to its interaction with the rotating blades requires the use of an average disk velocity. ${ }^{45}$ It will be evaluated from LES by averaging over the disk region, and over some period in time, yielding a velocity denoted by $\left\langle\bar{u}^{T}\right\rangle_{d}$. Then, the total thrust force can be written as

$$
F_{t}=-\rho_{2} \frac{1}{2} C_{T}^{\prime}\left\langle\bar{u}^{T}\right\rangle_{d}^{2} \frac{\pi}{4} D^{2},
$$

with the subscript $d$ denoting an averaging over the turbine disk region and the superscript $T$ denoting time filtering or averaging over a time scale on the order of $T$. Thus $\left\langle\bar{u}^{T}\right\rangle_{d}$ is the disk averaged and time-filtered velocity (further discussed below). Also, we define

$$
C_{T}^{\prime}=\frac{C_{T}}{(1-a)^{2}} .
$$

For the Betz limit $^{2}$ (i.e., $C_{T}=8 / 9$, and $a=1 / 3$ ), we obtain $C_{T}^{\prime}=2$. Using typical values $C_{T}=0.75$, and $a=1 / 4$, which may be found in existing wind turbines, ${ }^{2}$ and prior LES study ${ }^{43}$ leads to $C_{T}^{\prime}=4 / 3$. In the current study, we perform LES using various $C_{T}^{\prime}$ values around a baseline case with $C_{T}^{\prime}=4 / 3$, as will be further discussed in Sec. III C. Similar to the definition of $c_{\mathrm{ft}}$ [cf. Eq. (9)], we also introduce $c_{\mathrm{ft}}^{\prime}=\pi C_{T}^{\prime} /\left(4 s_{x} s_{y}\right)$.

In the JHU-LES code and the KULeuven code, two slightly different approaches are followed for the implementation of the wind-turbine forces $F_{t}$ and the disk-averaged local velocity $\left\langle\bar{u}^{T}\right\rangle_{d}$. In the JHU-LES code the total force $F_{t}$ is distributed among all grid points that fall into the windturbine disk region. This region is a circle with diameter $D$ and centered at particular locations in which wind turbines are located. In the streamwise direction, the rotor disk region is taken as a single grid point, i.e., in a mesh region of length $\Delta x=L_{x} / N_{x}$, where $L_{x}$ is the streamwise domain length and $N_{x}$ is the number of grid points in that direction. The force is distributed proportional to the computational mesh frontal area that coincides with the wind-turbine disk, i.e., $\gamma_{j, k} \Delta y \Delta z$, where $\gamma_{j, k}$ is the fraction of area overlap between the cell at grid point $(j, k)$ (positions $y_{j}, z_{k}$ ) and the circle. For the interior grid points representing meshes entirely contained inside the circle, the factor is $\gamma_{j, k}=1$. For grid points representing meshes entirely outside the circle, the factor is $\gamma_{j, k}=0$. For cells with partial overlap, the factor corresponds to the fractional area overlap.

Then, the force (per unit mass) in the streamwise direction at a given grid point $(i, j, k)$ corresponding to positions $\left(x_{i}, y_{j}, z_{k}\right)$ is given by

$$
f_{1}\left(x_{i}, y_{j}, z_{k}\right)=-\frac{1}{2} C_{T}^{\prime}\left\langle\bar{u}^{T}\right\rangle_{d}^{2} \frac{\gamma_{j, k}}{\Delta x} .
$$

Finally, in the JHU-LES code, the disk-averaged local velocity $\left\langle\bar{u}^{T}\right\rangle_{d}$ is evaluated by spatial averaging over all grid points in the wind-turbine disk, and temporal averaging 
TABLE I. Summarizing parameters of the various LES cases. Between brackets is indicated which code is used: "L" refers to the KULeuven code and "J" refers to the JHU-LES code.

\begin{tabular}{lccccccccc}
\hline \hline & $s_{x} / s_{y}$ & $s_{x}$ & $4 s_{x} s_{y} / \pi$ & $N_{t}$ & $L_{x} \times L_{y} \times H$ & $N_{x} \times N_{y} \times N_{z}$ & $z_{0, \mathrm{lo}}$ & $C_{T}^{\prime}$ & $c_{\mathrm{ft}}^{\prime}$ \\
\hline A1 (L) & 1.5 & 7.85 & 52.36 & $4 \times 6$ & $\pi \times \pi \times 1$ & $128^{3}$ & $10^{-4}$ & 1.33 & 0.025 \\
A2 (J) & 1.5 & 7.85 & 52.36 & $4 \times 6$ & $\pi \times \pi \times 1$ & $128^{3}$ & $10^{-4}$ & 1.33 & 0.025 \\
A3 (L) & 1.5 & 7.85 & 52.36 & $8 \times 6$ & $2 \pi \times \pi \times 1$ & $128 \times 192 \times 61$ & $10^{-4}$ & 1.33 & 0.025 \\
A4 (L) & 1.5 & 7.85 & 52.36 & $8 \times 6$ & $2 \pi \times \pi \times 1.5$ & $128 \times 192 \times 92$ & $10^{-4}$ & 1.33 & 0.025 \\
B (J) & 1.5 & 7.85 & 52.36 & $4 \times 6$ & $\pi \times \pi \times 1$ & $128^{3}$ & $10^{-4}$ & 2.00 & 0.038 \\
C (J) & 1.5 & 7.85 & 52.36 & $4 \times 6$ & $\pi \times \pi \times 1$ & $128^{3}$ & $10^{-4}$ & 0.60 & 0.012 \\
D (J) & 1.5 & 7.85 & 52.36 & $4 \times 6$ & $\pi \times \pi \times 1$ & $128^{3}$ & $10^{-3}$ & 1.33 & 0.025 \\
E (J) & 1.5 & 7.85 & 52.36 & $4 \times 6$ & $\pi \times \pi \times 1$ & $128^{3}$ & $10^{-5}$ & 1.33 & 0.025 \\
F (J) & 1.5 & 7.85 & 52.36 & $4 \times 6$ & $\pi \times \pi \times 1$ & $128^{3}$ & $10^{-6}$ & 1.33 & 0.025 \\
G (L) & 1.5 & 15.7 & 209.4 & $4 \times 3$ & $2 \pi \times \pi \times 1$ & $128 \times 192 \times 61$ & $10^{-4}$ & 1.33 & 0.0064 \\
H (L) & 1.5 & 6.28 & 33.51 & $10 \times 8$ & $2 \pi \times 1.07 \pi \times 1$ & $128 \times 192 \times 57$ & $10^{-4}$ & 1.33 & 0.040 \\
I (L) & 1.5 & 5.24 & 23.27 & $12 \times 9$ & $2 \pi \times \pi \times 1$ & $128 \times 192 \times 61$ & $10^{-4}$ & 1.33 & 0.057 \\
J (L) & 2 & 9.07 & 52.36 & $7 \times 7$ & $2.02 \pi \times 1.01 \pi \times 1$ & $128 \times 192 \times 61$ & $10^{-4}$ & 1.33 & 0.025 \\
K (L) & 1 & 6.41 & 52.36 & $10 \times 5$ & $2.04 \pi \times 1.02 \pi \times 1$ & $128 \times 192 \times 60$ & $10^{-4}$ & 1.33 & 0.025 \\
\hline \hline
\end{tabular}

over a time window of $T u_{*} / H=0.27$ (corresponding to $T=10 \mathrm{~min}$ using possible reference dimensional values $u_{*}=0.45 \mathrm{~m} / \mathrm{s}$ and $\left.H=1000 \mathrm{~m}\right)$. The time averaging is done using a one-sided exponential time filter (first-order relaxation process).

The KULeuven code contains an alternative drag-disk force implementation, which more easily allows to change the positioning and orientation of turbines. In a first step, turbine forces are described in the turbine-rotor plane. In a second step, these forces are filtered using a Gaussian convolution filter on locations which correspond to the coordinates of the LES grid. To further detail this procedure, we introduce the coordinate system $\mathbf{y}=\left(y_{1}, y_{2}\right)$ in the turbine rotor plane, with origin in the rotor center, $y_{1}$ the horizontal, and $y_{2}$ the vertical directions. In the LES coordinate frame the rotor plane is given by coordinates $\mathbf{x}_{\mathbf{t}}$ which satisfy $\mathbf{e}_{\perp} \cdot\left(\mathbf{x}_{\mathbf{t}}-\mathbf{x}_{\mathbf{0}}\right)=0$ with $\mathbf{x}_{\mathbf{0}}$ as the coordinate of the rotor center and $\mathbf{e}_{\perp}$ a unit vector perpendicular to the rotor plane. Take $f_{t, i}(\mathbf{y}) \quad(i=1,2,3)$ the turbine-forces per unit mass in the turbine-rotor coordinate frame, then the filtered forces in the LES coordinate frame correspond to

$$
f_{i}(\mathbf{x})=Q_{i j} \mathcal{F}_{t, j}(\mathbf{x}),
$$

where $Q_{i j}$ is a rotation matrix, accounting for possible different orientations between the $\mathbf{y}$, and $\mathbf{x}$ coordinate frames, and $\mathcal{F}_{t, i}$ are the filtered turbine forces at the LES-grid locations $\mathbf{x}$, i.e.,

$$
\begin{aligned}
\mathcal{F}_{t, i}(\mathbf{x})= & \iiint G\left(\mathbf{x}-\mathbf{x}^{\prime}\right) \delta\left(\mathbf{e}_{\perp} \cdot\left(\mathbf{x}^{\prime}-\mathbf{x}_{\mathbf{0}}\right)\right) \\
& \times f_{t, i}\left(\mathbf{y}\left(\mathbf{x}^{\prime}-\mathbf{x}_{\mathbf{0}}\right)\right) \mathrm{d}^{3} \mathbf{x}^{\prime},
\end{aligned}
$$

where $\delta(x)$ is the Dirac function. We select a Gaussian filter, with filter kernel $G(\mathbf{x})=\left[6 /\left(\pi \Delta^{2}\right)\right]^{3 / 2} \exp \left(-6\|\mathbf{x}\|^{2} / \Delta^{2}\right)$, where $\Delta=1.5 h$ (and $h$ is the grid spacing) is used to avoid Gibbs oscillations on the LES grid. A similar smoothing approach was used for actuator-line representations in Ref. 10.

In the current study, turbines are always oriented perpendicular to the streamwise direction $x_{1}$, such that $Q_{i j}$ is an identity matrix. Further, the turbine forces only include thrust effects, which are distributed constant over the turbine rotor disk with diameter $D$. Consequently, $f_{2}=f_{3}=0$ and $f_{1}=\mathcal{R}_{t}(\mathbf{x}) f_{t, 1}$, with $\rho f_{t, 1} \pi D^{2} / 4=F_{t}[$ cf. Eq. (18)], and where

$$
\begin{aligned}
\mathcal{R}(\mathbf{x})= & \iiint G\left(\mathbf{x}-\mathbf{x}^{\prime}\right) \delta\left(\mathbf{e}_{\perp} \cdot\left(\mathbf{x}^{\prime}-\mathbf{x}_{\mathbf{0}}\right)\right) \\
& \times \mathrm{H}\left(D / 2-\left\|\mathbf{y}\left(\mathbf{x}^{\prime}-\mathbf{x}_{\mathbf{0}}\right)\right\|\right) \mathrm{d}^{3} \mathbf{x}^{\prime}
\end{aligned}
$$

is the geometrical representation of the rotor disk smoothed out into the LES domain using a Gaussian filter [and $\mathrm{H}(x)$ is the Heaviside function].

In order to determine the disk averaged local velocity $\langle u\rangle_{d}$ needed for the determination of the thrust force $F_{t}$, we employ (in the KULeuven code) the geometrical rotor footprint $\mathcal{R}(\mathbf{x})$ as a weighting function for the averaging such that

$$
\langle u\rangle_{d}=\frac{4}{\pi D^{2}} \iiint \mathcal{R}(\mathbf{x}) \widetilde{\mathbf{u}}(\mathbf{x}) \cdot \mathbf{e}_{\perp} \mathrm{d}^{3} \mathbf{x} .
$$

Further, as in the JHU-LES code, $\left\langle\bar{u}^{T}\right\rangle_{d}$ is obtained from $\langle u\rangle_{d}$ by using a one-sided exponential time-filter using a time window of $T u_{*} / H=0.6$.

\section{Suite of LES cases}

In the various simulations, we vary the resolution (given by the parameters $L_{x}, L_{y}, H$ and $N_{x}, N_{y}, N_{z}$ ), the number of wind turbines $\left(N_{t}\right)$, the distances among them $\left(s_{x} D\right.$ and $\left.s_{y} D\right)$, and the relative sparseness of their distribution $\left(S / A=4 s_{x} s_{y} / \pi\right)$, the thrust coefficient $C_{T}^{\prime}$ that represents the loading of the wind turbine, and the bottom surface roughness scale $z_{0,10}$. The grid spacing is uniform in all three directions. The suite of LES cases is described in Table I.

As can be appreciated from the table, the simulations are divided among the JHU-LES and KULeuven codes. Simulations with the KULeuven code focus more on variations in turbine arrangements, with different $s_{x}-s_{y}$ combinations 
(a)

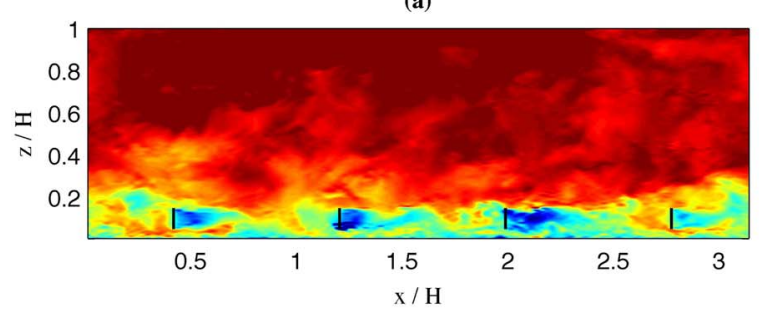

(b)

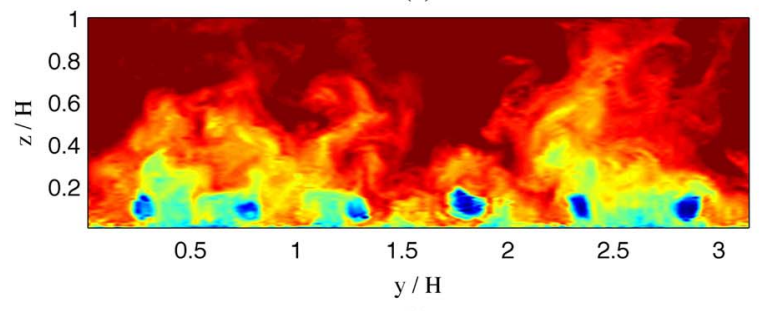

(c)

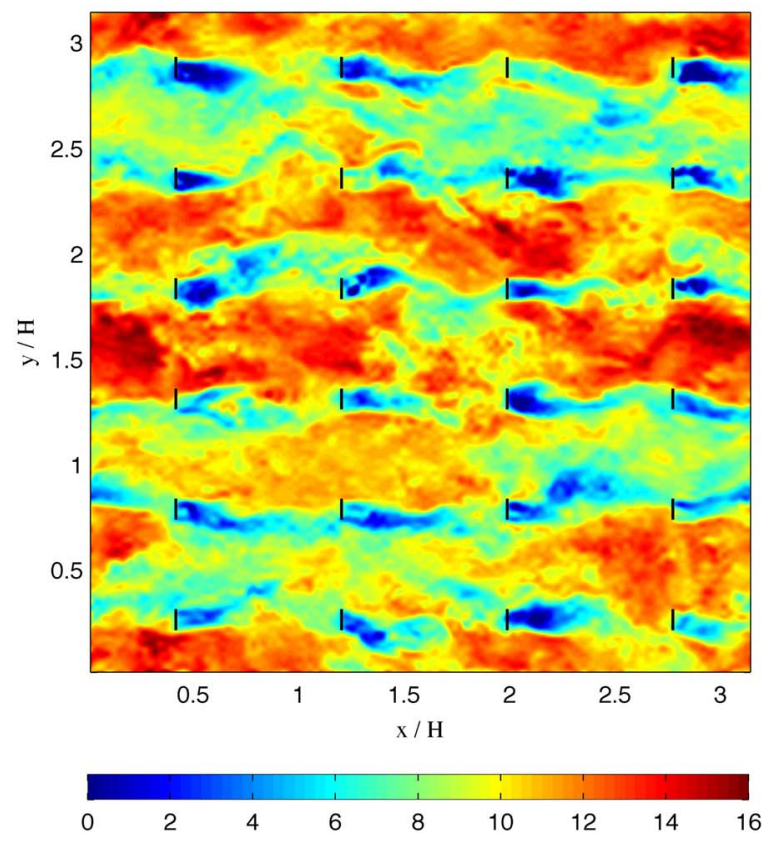

FIG. 1. (Color online) Instantaneous contours of streamwise velocity from LES of a fully developed WTABL (baseline case A2); (a) on a $x$ - $z$ plane cutting through the middle of a column of wind turbines. (The location of the wind turbine disks are indicated with vertical black lines). (b) On a cross-stream $y$ - $z$ plane at a distance $\sim 1 D$ downstream of a row of wind turbines. (c) On a $x-y$ plane at a height $z$ corresponding to hub height (the wind turbine centers).

since this is the more flexible code in terms of turbine placement on the LES grid (cf. Sec. III B). Simulations with the JHU-LES code mainly focus on $z_{0, \text { lo }}$ variations since the scale-dependent Lagrangian dynamic model employed by this code is expected to better describe the flow and roughness effects near the bottom surface (cf. Sec. III A). All simulations use $C_{T}^{\prime}=1.33$, except cases $\mathrm{B}$ and $\mathrm{C}$, where a higher value $C_{T}^{\prime}=2.0$ (corresponding to the Betz limit), and a lower value $C_{T}^{\prime}=0.6$ are considered. Finally, the reference case (using $s_{x} / s_{y}=1.5,4 s_{x} s_{y} / \pi=52.36, \quad z_{0,10}=10^{-4}$, and $C_{T}^{\prime}=1.33$ ) is simulated four times (cases $\mathrm{A} 1, \mathrm{~A} 2, \mathrm{~A} 3$, and A4), which allows us to assess the effect of different codes and different computational domains.

All simulations are initialized with a startup run of 60
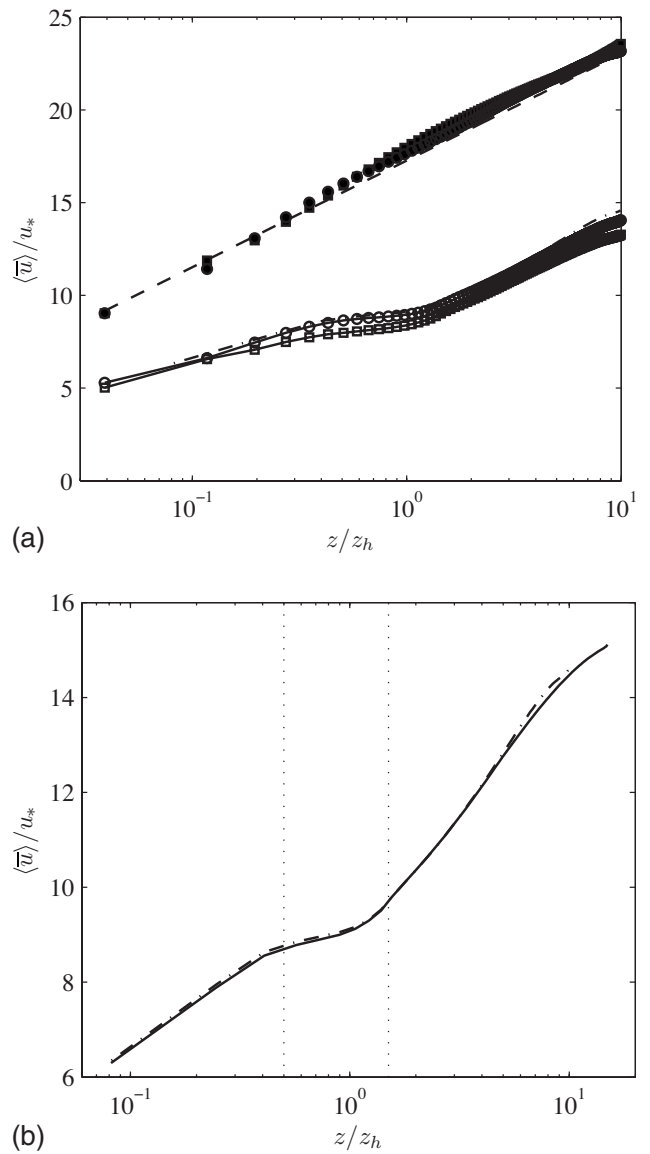

FIG. 2. (a) Mean velocity profile of the baseline cases A1 (-O), A2 ( $\square$ ), and A3 (dash-dotted line), comparing the effects of slightly different numerical implementation of the LES, subgrid models, drag-disk force implementation, and domain and grid selections. The top dashed line is $\kappa^{-1} \ln \left(z / z_{0,10}\right)$, the log-law behavior expected without wind turbine models; $(\bullet, \square)$ cases A1 and A2 without turbine loads. (b) Mean velocity profile of cases A3 (dash-dotted line) and A4 (full line). The vertical dotted lines mark the bottom and top of the turbine-rotor planes.

nondimensional time units (where the dimensionless time is in units of $H / u_{*}$ ), which was found sufficiently long to achieve statistical stationarity. Subsequently, statistics are accumulated over $60(\mathrm{~L})$ and $15(\mathrm{~J})$ nondimensional time units.

While the number of turbines (e.g., $4 \times 6)$ and the size of the domain are not sufficient by themselves to lead to a "fully developed condition" (cf. discussion in Sec. I), in combination with the periodic boundary conditions in the horizontal plane and the very long simulation time, the simulated flow conditions can be considered effectively fully developed.

A sample of instantaneous results is shown in Figs. 1(a)-1(c). They are contour plots of streamwise velocity in three perpendicular planes. The location of the wind-turbine disks are indicated with black lines. As seen in Fig. 1(a), which shows $x-z$ plane cutting through the middle of a column of wind turbines, the slow-moving fluid in the wakes recovers significantly before reaching the next turbines. Internal boundary-layer type structures above the wind turbines are also visible. Figure 1(b) shows instantaneous cuts through a cross-stream $y-z$ plane at a distance $\sim 1 D$ downstream of a row of wind turbines. The array has significant 


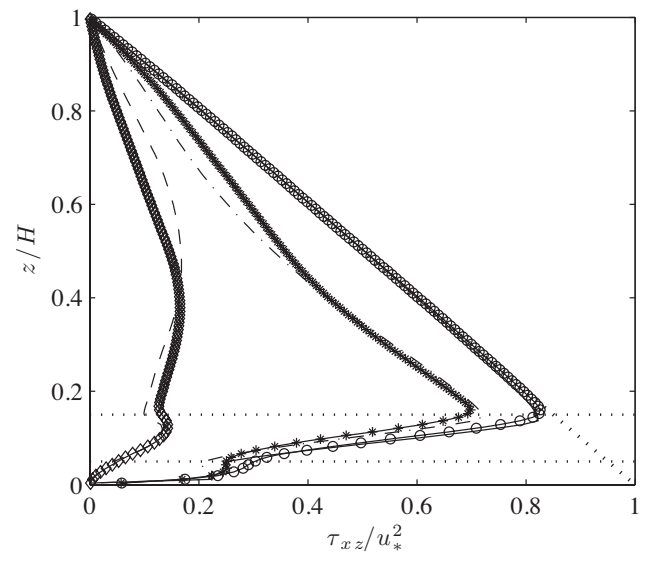

FIG. 3. Vertical profiles of shear stresses of the baseline cases A1 and A2. The Reynolds shear stresses are indicated using dot-dashed line (A2) and asterisks (A1), while the dispersive stresses are denoted with dashed line (A2) and open diamonds (A1). Their respective sum is shown by a solid line (A2) and open circles (A1).

effects even on the high regions of the boundary layer, where strong ejection and sweep motions are visible. The horizontal cut shown in Fig. 1(c), $x-y$ plane, is also through the windturbine centers. Here, the significant meandering of windturbine wakes is clearly visible. It is well known that such meandering increases significantly the loads on wind-turbine structures and causes additional (generally unwanted) variability. ${ }^{9}$

\section{VERTICAL PROFILES OF HORIZONTAL AVERAGES}

Figure 2(a) shows the mean velocity profile of the baseline cases A1, A2, and A3, comparing the effects of slightly different numerical implementation of the LES, subgrid model, drag-disk force implementation, and effects of domain and grid selection. The velocity is normalized with the imposed pressure-gradient based parameter $u_{*}$. Henceforth, $u_{*}$ and $u_{* \mathrm{hi}}$ used in the prior sections will be used interchangeably. Also shown in the figure are the results from LES without any wind turbines present, showing agreement with the log-law behavior near the surface. As can be appreciated, the effects of the computational domain and grid (compare cases A1 and A3) are small. Differences between the two LES codes (compare cases A1 and A2) are a bit more pronounced; nevertheless, the profiles compare quite well. There are some minor differences near the ground surface. There, the Smagorinsky model results in slightly higher velocity gradient (and thus a slightly higher velocity at the turbine height), compared to the scale-dependent model. The differences near the wind-turbine region may also be due to the small differences in the implementation of the drag force and how it is distributed among the grid points. As a result, there are some differences also at $z / H=1$, reflecting small differences in the overall effective roughness characteristics computed from cases A1-A3. We believe this level of difference is a useful indication of the level of differences to be expected from modeling implementations in a complex flow simulation.

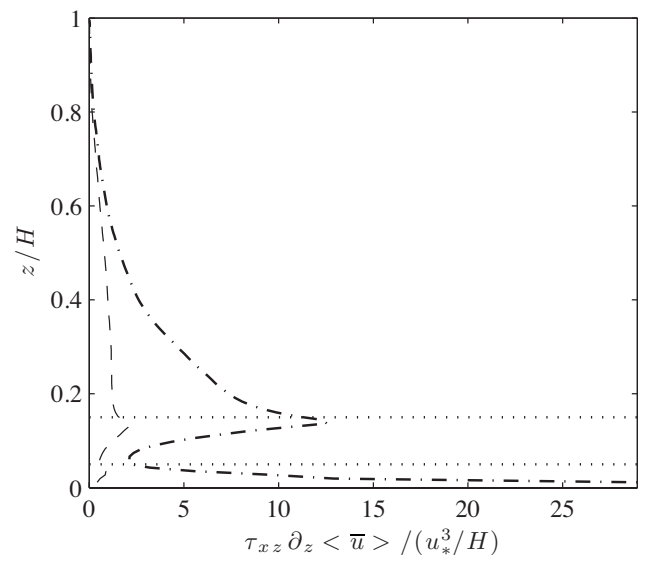

FIG. 4. Vertical profiles of dissipation of mean kinetic energy or the baseline case A2 due to turbulent Reynolds stresses (dot-dashed line; also production of turbulent kinetic energy) and due to dispersive stresses (dashed line).

In Fig. 2(b), mean velocity profiles from cases A3 and A4 are presented, comparing the effect of a different height of the ABL. Case A4 consists of the same turbine loading, and pressure gradient as case A3, but with a height of $H=1.5$. Hence, the imposed pressure-gradient based parameter $u_{*}$ is $(1.5)^{1 / 2}$ higher for case A4. The mean velocity profiles in Fig. 2(b) show very good agreement when normalized using inner scales $u_{*}$, and $z_{h}$, and profiles match up to a height of approximately $5 z_{h}$. This supports our hypothesis that the wind turbines mainly interact with the inner region of the boundary layer, and that results are independent of the outer-region effects such as boundary layer thickness, etc. (cf. discussion in Sec. I).

The Reynolds shear stress $-\left\langle\overline{u^{\prime} w^{\prime}}\right\rangle$ and dispersive shear stress profiles $-\left\langle\bar{u}^{\prime \prime} \bar{w}^{\prime \prime}\right\rangle$ of the baseline runs $\mathrm{A} 1$ and $\mathrm{A} 2$ are shown in Fig. 3. The results are shown as lines (case A2, JHU code) and symbols (case A1, KULeuven code). The Reynolds shear stresses are larger, but the dispersive stresses cannot be neglected. They range between $15 \%$ and $35 \%$ of the stress depending on height. Their sum is shown as solid lines (JHU code) and hollow circles (KULeuven code) and shows the expected linear behavior until close to the top of the wind-turbine region (delimited by the horizontal dotted lines between $z / H=0.05$ and $z / H=0.15)$. In this region, the drag due to the turbines begins to deplete the momentum fluxes until below the wind-turbine region. Close to the ground, the subgrid stress provides the remaining momentum transport.

Next, we study the vertical structure of the flow from the point of view of kinetic energy [see Eq. (3)]. Figure 4 shows the profiles of kinetic energy dissipation caused by the turbulence $\left(-\left\langle\overline{u^{\prime} w^{\prime}}\right\rangle \partial\langle\bar{u}\rangle / \partial z\right)$ and by the dispersive stresses $\left(-\left\langle\bar{u}^{\prime \prime} \bar{w}^{\prime \prime}\right\rangle \partial[\langle\bar{u}\rangle] / \partial z\right)$. This is the rate at which kinetic energy of the horizontally averaged mean flow is being lost due to production of turbulent kinetic energy and due to mixing of spatial variations in the time-averaged mean velocity distribution, respectively. They do not necessarily need to be positive, especially the latter. Without wind turbines the maximum generation of turbulent kinetic energy takes place very near the ground and decreases rapidly with increasing 


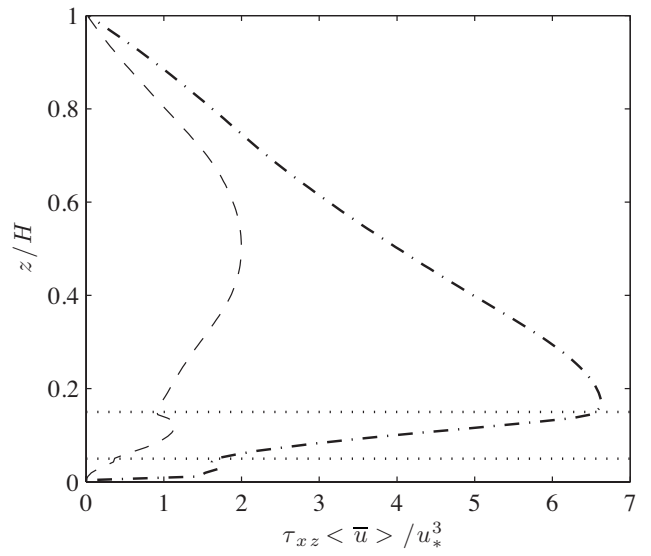

FIG. 5. Vertical profiles of fluxes of kinetic energy for the baseline case A2 due to turbulent Reynolds stresses (dot-dashed line) and due to dispersive stresses (dashed line).

height. When introducing wind turbines a second peak occurs at the top of the wind-turbine region, where large turbulence production is due to the peak of Reynolds stresses there.

Figure 5 shows the profiles of flux of kinetic energy. The dispersive fluxes, $-\left\langle\bar{u}^{\prime \prime} \bar{w}^{\prime \prime}\right\rangle\langle\bar{u}\rangle$, are associated with the mean velocity's spatial variations and include effects coming from wake recovery, etc. As can be seen, in this case they are, on the average, smaller (but not negligible) when compared to the kinetic energy fluxes caused by the turbulence, $-\left\langle\overline{u^{\prime} w^{\prime}}\right\rangle\langle\bar{u}\rangle$. It is of interest to compare these results with the mechanical power extracted by the wind turbines.

In recent wind tunnel experiments ${ }^{46}$ that measured directly the power extracted by model wind turbines in a $3 \times 3$ model wind farm, velocity field measurements showed that the vertical fluxes of kinetic energy were of the same order of magnitude as the power extracted by the wind turbines. A similar analysis can be done based on the LES results. For this purpose, we integrate Eq. (3) between $z_{h}-D / 2$ and $z_{h}+D / 2$ and obtain

$$
\mathcal{W}_{p}+\Phi\left(z_{h}+D / 2\right)-\Phi\left(z_{h}-D / 2\right)=\mathcal{D}+\mathcal{W}_{T},
$$

where

$$
\mathcal{W}_{p}=-\frac{D}{\rho}\langle\bar{u}\rangle_{D} \frac{d p_{\infty}}{d x}
$$

is the forcing power due to the applied pressure gradient in the wind-turbine region,

$$
\Phi(z)=-\left(\left\langle\overline{u^{\prime} w^{\prime}}\right\rangle\langle\bar{u}\rangle+\left\langle\bar{u}^{\prime \prime} \bar{w}^{\prime \prime}\right\rangle\langle\bar{u}\rangle\right)
$$

is the total flux of kinetic energy by turbulence and dispersive stresses, and

$$
\mathcal{D}=\int_{z_{h}-D / 2}^{z_{h}+D / 2}-\left(\left\langle\overline{u^{\prime} w^{\prime}}\right\rangle+\left\langle\bar{u}^{\prime \prime} \bar{w}^{\prime \prime}\right\rangle\right) \frac{\partial\langle\bar{u}\rangle}{\partial z} d z
$$

is the dissipation term integrated over the wind-turbine area. The last term $\mathcal{W}_{T}$ is defined as

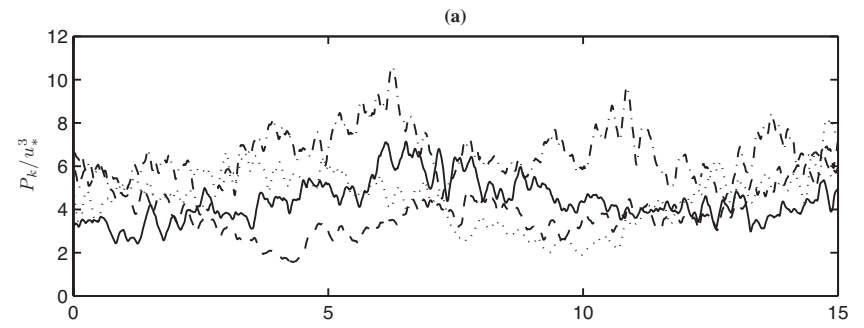

(b)

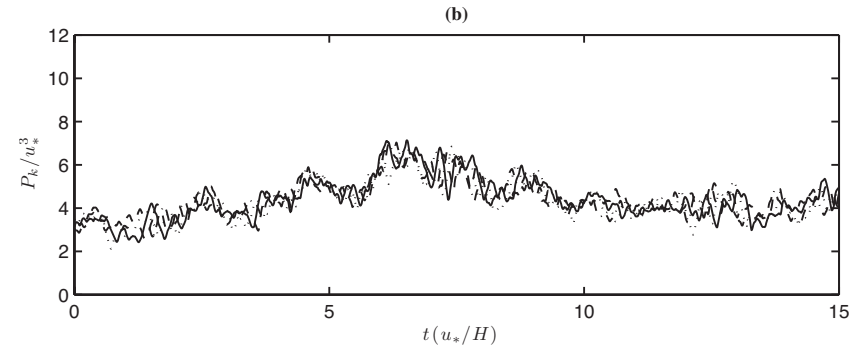

FIG. 6. Extracted power density by four different turbines in simulation A2. In (a) four different WTs corresponding to the same row, but different columns: $(\mathrm{col}=1$, row $=1)$ solid line, $(c 2, r 1)$ dashed line, $(c 3, r 1)$ dotted line and $(c 5, r 1)$ dot-dashed line. In (b) four different WTs all aligned in the same column, but from different rows: $(\mathrm{col}=1$, row $=1)$ solid line, $(c 1, r 2)$ dashed line, $(c 1, r 3)$ dot-dashed line and $(c 1, r 4)$ dotted line.

$$
\mathcal{W}_{T}=-\langle\bar{u}\rangle_{D} \frac{\overline{F_{t}} / \rho}{s_{x} s_{y} D^{2}},
$$

where $\langle\bar{u}\rangle_{D}$ is the time and horizontally averaged mean flow velocity $\langle\bar{u}\rangle$, also averaged over the WT's vertical layer. This is subtly different from the power "extracted" by the windturbine model, which is obtained by time averaging the mechanical power extracted according to

$$
\overline{\mathcal{P}}_{T}=\frac{1}{N_{t k=1}} \sum_{t}^{N_{t}} \bar{P}_{k}, \quad \text { where } P_{k}=\left(\frac{-F_{t}\left\langle\bar{u}^{T}\right\rangle_{d}}{\rho s_{x} s_{y} D^{2}}\right)_{k}
$$

is the instantaneous power extraction, and $\overline{\mathcal{P}}_{T}$ includes additional averaging over the number of wind turbines $N_{t}$ in the computational domain $\left(N_{t}=24\right.$ for case A2). Figure 6 shows the time series for $P_{k}$ for several of the wind turbines. The four turbines shown in the top Fig. 6(a) are in different columns, i.e., they are not downstream from each other. There is significant spatial variability of the extracted power between WTs of different columns. This is consistent with the significant spatial variations of the velocity field seen in the visualizations in Fig. 1. Conversely, the wind turbines in the same column (downstream of each other) shown in Fig. 6(b) are highly correlated. The time average value over all wind turbines has been evaluated and is approximately $\overline{\mathcal{P}}_{T} / u_{*}^{3} \approx 4.93$. Next, we compare this value with the terms in the horizontally averaged energy equation discussed above.

From Fig. 5, it is evident that the difference in the kinetic energy fluxes between the top and bottom heights of the turbine region is $\delta \Phi / u_{*}^{3} \sim 5.4$. To evaluate $\mathcal{W}_{p}$, we note that the mean velocity in the wind-turbine region (from integrating the mean velocity in the wind-turbine region) is $\langle\bar{u}\rangle_{D} / u_{*} \sim 8.5$. Therefore, the pressure forcing term is $\mathcal{W}_{p} / u_{*}^{3} \approx D / H \times 8.5 \approx 0.85$. From Fig. 4 and by integrating Eq. (28), a value $\mathcal{D} / u_{*}^{3} \sim 0.68$ is obtained for the dissipation 
of mean kinetic energy [we remark that $\mathcal{W}_{T}$ is also evaluated and results in $\mathcal{W}_{T} \sim 5.7 u_{*}^{3}$, which as would be expected closes the power budget (to within $2 \%$, the remaining variation being attributed to slight lack of full statistical convergence)]. Note that $\mathcal{W}_{T}$ differs from $\overline{\mathcal{P}}_{T}$ since $\left.\langle\bar{u}\rangle_{D}\right\rangle\left\langle\bar{u}^{T}\right\rangle_{d}$. The difference in power terms is associated with the kinetic energy associated with spatial inhomogeneity of the mean flow in the horizontal direction.

The most interesting comparison to make is between the power extracted at the wind turbines $\overline{\mathcal{P}}_{T}$ and the vertical flux of kinetic energy $\Phi$. As can be appreciated, they are of the same order of magnitude, $\overline{\mathcal{P}}_{T}$ being about $90 \%$ of $\delta \Phi$ (and $75 \%$ of $\Phi$ on top of the wind turbine). From these numbers, it is clear that in the fully developed WTABL, the way the kinetic energy "arrives" at the wind turbines is by means of turbulence-facilitated vertical fluxes.

A further remark is needed on the relationship between the computed $\overline{\mathcal{P}}_{T}$ and the mechanical power available at the shaft on a real wind turbine. The mechanical power which can be converted to electricity rather must be expressed using the power coefficient $C_{P}$ (or $C_{P}^{\prime}$ when using the disk velocities instead of $U_{\infty}$ ). Only in the absence of friction does $C_{P}^{\prime}$ correspond to $C_{T}^{\prime}$ (with a maximum corresponding the Betz limit, ${ }^{2}$ in which case $C_{T}^{\prime}=C_{p}^{\prime}=2.0$, which corresponds to the well-known values $C_{P}=16 / 27$ and $C_{T}=8 / 9$ ). In reality, the drag on the turbine blades plays an important role, which mainly effects the torque, such that $C_{P}^{\prime}$ is in practice significantly lower then $C_{T}^{\prime}$ (cf. Ref. 45). The losses related to these drag effects result into small-scale turbulence in the wake of the turbine blades (typically situated in the subgrid scales of our LES), which is eventually dissipated into heat.

\section{MEASURED EFFECTIVE ROUGHNESS AND FRICTION VELOCITY}

In this section we evaluate the effective roughness length $z_{0, \text { hi }}$ for all the cases simulated to help develop parametrizations that may be used in very large-scale simulations of wind-turbine arrays. First, Figs. 7(a)-7(c) show a collection of different mean velocity profiles for several of the cases with different geometrical loading $4 s_{x} s_{y} / \pi$ [Fig. 7(a)], aspect ratios of the turbine placement $s_{x} / s_{y}$ [Fig. 7(b)], and different ground roughness [Fig. 7(c)]. Also shown are log-law approximations through a region above and below the wind turbines. The roughness length $\left(z_{0, \mathrm{hi}}\right)$ is obtained from the measured mean velocity profile shapes above the windturbine region. As can be seen in Fig. 7, a good logarithmic behavior is visible there in most of the cases. Then good results for $z_{0, \text { hi }}$ can be obtained simply by using a single velocity value at a height of $2 z_{h}=0.2 H$. The expression used is $\langle\bar{u}\rangle\left(2 z_{h}\right)=(1 / \kappa) \log \left(2 z_{h} / z_{0, \text { hi }}\right)$, where $\langle\bar{u}\rangle\left(2 z_{h}\right)$ is obtained from the LES and $z_{0, \text { hi }}$ is obtained by solving this equation using $\kappa=0.4$. The results for the roughness $z_{0, \mathrm{hi}}$ are also reported in Table II. Attempts to fit the results using the additional parameter of a "displacement height" did not lead to meaningful improvements. We conclude that for wind farms (unlike dense plant canopies) if a displacement length exists, it will tend to be very small.
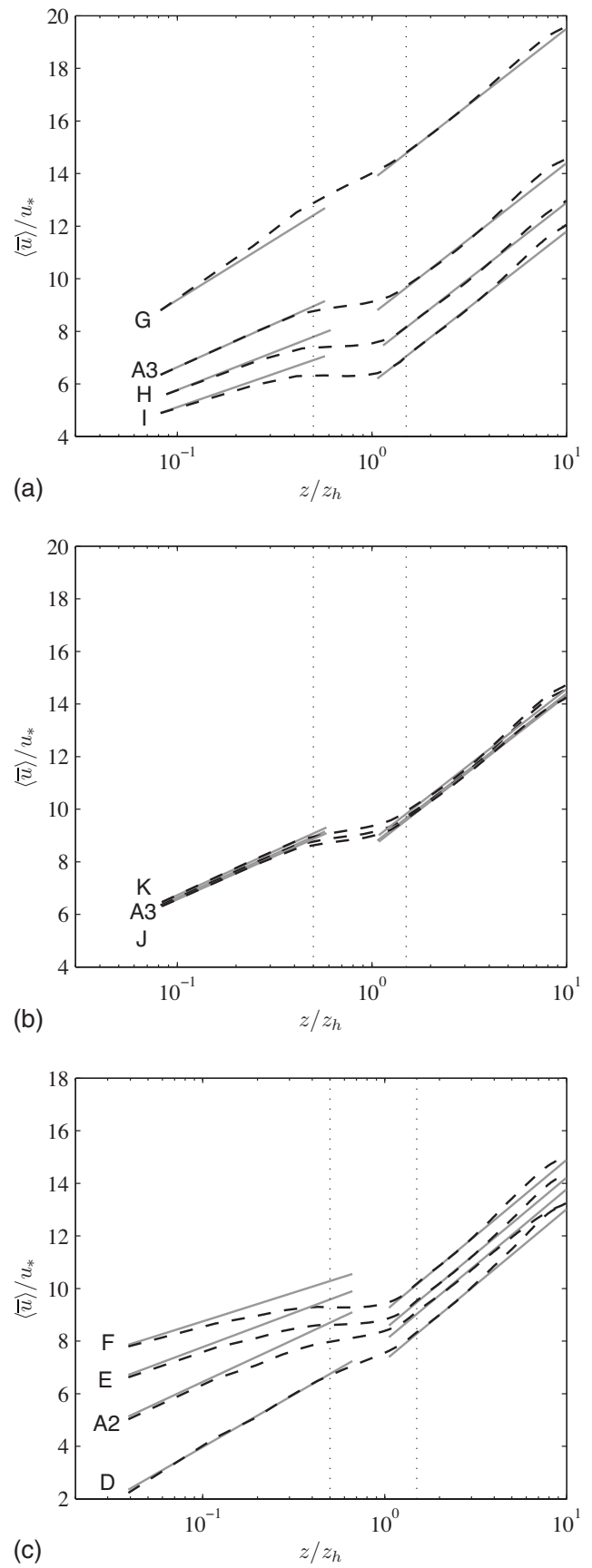

FIG. 7. Mean velocity profiles for wind farms with different parameters. (a) Results for varying geometrical loading, with $4 s_{x} s_{y} / \pi \approx 209$ (case G), $4 s_{x} s_{y} / \pi \approx 52$ (case A3), $4 s_{x} s_{y} / \pi \approx 33$ (case H), and $4 s_{x} s_{y} / \pi \approx 23$ (case I). (b) Results with varying aspect ratios $s_{x} / s_{y}=2$ (case J), $s_{x} / s_{y}=1.5$ (case A3), and $s_{x} / s_{y}=1$ (case K). (c) Simulations results for different surface roughnesses $z_{0,10}=10^{-3}$ (case D), $z_{0,10}=10^{-4}$ (case A2), $z_{0,10}=10^{-5}$ (case E), and $z_{0,10}=10^{-6}$ (case F). In gray: log profiles; below the turbines (near the bottom): $\langle\bar{u}\rangle / u_{* l_{0}}=\log \left(z / z_{0, l_{0}}\right) / \kappa$; above the turbines: $\langle\bar{u}\rangle / u_{* \mathrm{hi}}=\log \left(z / z_{0, \mathrm{hi}}\right) / \kappa$ (values for $u_{* \text { *o }_{\mathrm{o}}}$ and $z_{0, \mathrm{hi}}$ are listed in Table II). A detailed overview of the different cases is summarized in Table I.

From Fig. 7, it is appreciated that the geometrical loading, and the roughness of the ground surface have a dominant effect on the wind profiles in the boundary layer, and the effective roughness length $z_{0, \text { hi }}$ observed by the higher layers in the WTABL. Also the variation in $C_{T}^{\prime}$ (results not shown in the figure) has an important effect. The effects of the aspect ratio $s_{x} / s_{y}$ are an order of magnitude lower. The effect of 
TABLE II. Effective roughness heights and friction velocities as determined from LES for the different cases.

\begin{tabular}{lccc}
\hline \hline & $z_{0, \mathrm{hi}} / z_{h}$ & $u_{* \mathrm{lo}} / u_{*}$ & $u_{* \mathrm{lo}}^{t u} / u_{*}$ \\
\hline A1 (L) & $3.4 \times 10^{-2}$ & 0.58 & 0.55 \\
A2 (J) & $4.09 \times 10^{-2}$ & 0.56 & 0.51 \\
A3 (L) & $3.2 \times 10^{-2}$ & 0.58 & 0.54 \\
B (J) & $5.2 \times 10^{-2}$ & 0.54 & 0.47 \\
C (J) & $1.7 \times 10^{-2}$ & 0.66 & 0.60 \\
D (J) & $5.5 \times 10^{-2}$ & 0.69 & 0.63 \\
E (J) & $3.4 \times 10^{-2}$ & 0.45 & 0.39 \\
F (J) & $2.6 \times 10^{-2}$ & 0.38 & 0.33 \\
G (L) & $0.41 \times 10^{-2}$ & 0.80 & 0.73 \\
H (L) & $5.8 \times 10^{-2}$ & 0.50 & 0.47 \\
I (L) & $8.9 \times 10^{-2}$ & 0.44 & 0.42 \\
J (L) & $3.2 \times 10^{-2}$ & 0.57 & 0.53 \\
K (L) & $2.9 \times 10^{-2}$ & 0.58 & 0.54 \\
\hline \hline
\end{tabular}

$s_{x} / s_{y}$ on the power extracted from the WTABL also remains below $10 \%$. Even though these differences, and geometricalplacement optimizations, may be somewhat relevant from the point of view of practical siting of wind turbines within an array, we will focus more on variations in $z_{0,10}, 4 s_{x} s_{y} / \pi$, and $C_{T}^{\prime}$ for the remainder of this work since they have a much larger impact on $z_{0, \mathrm{hi}}$ and on the horizontally averaged structure of the flow.

Of considerable interest for further comparison with models for $z_{0, \text { hi }}$ in the next section, is also the value obtained for the friction velocity $u_{* \text { lo }}$ below the wind turbines. It is obtained based on the definition suggested in Sec. II B, namely, as $u_{* \mathrm{lo}_{\mathrm{o}}}=\sqrt{\left\langle\overline{\tau_{1 w}}\right\rangle}$, where $\left\langle\overline{\tau_{1 w}}\right\rangle$ is the time and plane averaged wall shear stress applied in the LES [see Eq. (14)]. The results are shown in Table II. For comparison, it is also of interest to report a friction velocity obtained from the total stress curves at the bottom of the wind-turbine region, i.e.,

$$
u_{* 1 \mathrm{o}}^{t u}=\sqrt{-\left(\left\langle\overline{u^{\prime} w^{\prime}}\right\rangle+\left\langle\bar{u}^{\prime \prime} \bar{w}^{\prime \prime}\right\rangle\right)_{z_{h}-D / 2}} .
$$

These stresses are readily obtained from the LES. For instance, in Fig. 8 the total shear stress, dispersive stress, and Reynolds stress profiles are shown for two of the cases ( $F$ and D). Large differences exist on the dispersive and Reynolds stresses. Their sum allows a consistent estimation of $u_{* 1 \mathrm{l}}^{t u}$, and the results are listed in detail in Table II. In general, we find that $u_{* 1 \mathrm{o}}^{t u} \approx u_{* \mathrm{lo}}$ based on the wall stress, to within about $10 \%-15 \%$ in most cases.

Using the values for the effective roughness $z_{0, \text { hi }}$ and the lower friction velocity $u_{* l_{0}}^{t u}$ in Table II, we now evaluate different theoretical models for the effective roughness in Sec. VI.

\section{DISCUSSION: COMPARISON WITH MODELS}

In this section we gauge the accuracy of the existing models for effective roughness heights described in Sec. II B associated with wind-turbine arrays. The predictions are evaluated using Eqs. (4) and (11) using the parameters appropriate for each case. The results of the comparison are presented in several forms. We show all results in compact

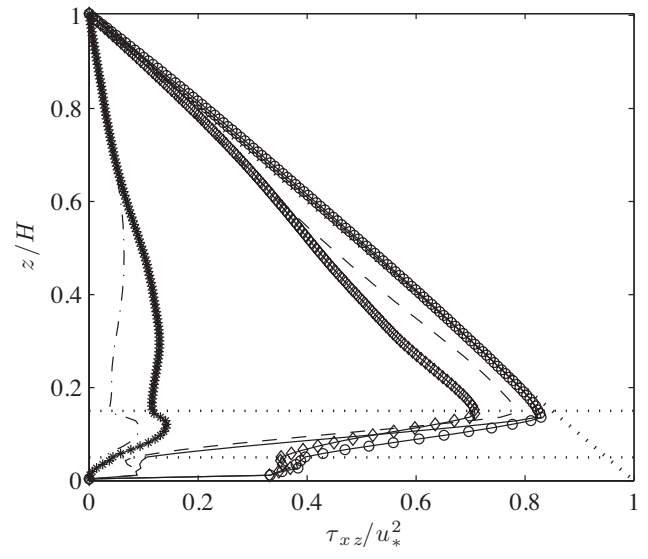

FIG. 8. Profiles of the total shear stress (case F in solid line and case D in open circles), dispersive stress (dot-dashed line for case F and asterisks for D), and Reynolds stress (dashed line for case F and open diamonds for case D).

form in Fig. 9 by plotting the ratio of model predicted roughness $\left(z_{0, \mathrm{mid}}^{\mathrm{mod}}\right)$ and the roughness obtained from LES ( $z_{0, \text { hi }}^{\mathrm{LES}}$, taken as the "correct" measured value). The results for each case are shown as function of the LES-predicted effective roughness.

It can be seen from this figure that the Lettau formula, which does not depend upon the bottom surface roughness nor the thrust coefficient (assuming one does not adjust the coefficient 1/2) is less accurate than the Frandsen formula, especially at high effective roughness lengths. However, even the Frandsen formula involves significant discrepancies (typically a factor of 2).

Based on the results of the simulation, a more accurate model may be suggested, in which the condition [Eq. (10)] is replaced by the assumption that there is a third (middle) logarithmic layer in between $z_{h}-D / 2$ and $z_{h}+D / 2$. For most of the LES cases the slope of the mean velocity profile there is almost zero. However, for the most lightly loaded WTABLs with sparse arrangements (e.g., case G) the slope is larger. The velocity at turbine height $z_{h}$ may be estimated by

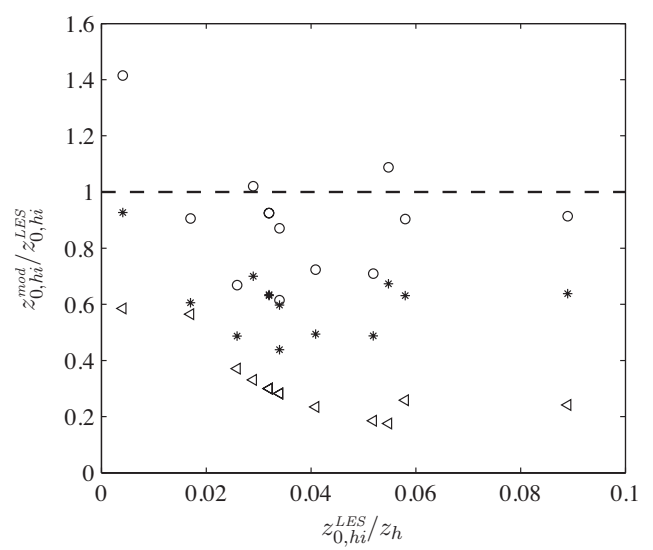

FIG. 9. Comparison between roughness heights obtained from models $\left(z_{0, \text { hi }}^{\text {mod }}\right)$ and those arising from LES $\left(z_{0, \text { hi }}^{\mathrm{LES}}\right)$. The comparison includes the Lettau formula (open triangles), the original Frandsen formula (stars), and the proposed modified formula (open circles) [see Eq. (39)]. 
taking the value below the turbine region and adding the logarithmic slope multiplied by the (logarithmic) distance to the wind-turbine height,

$$
\langle\bar{u}\rangle_{\mathrm{lo}}\left(z_{h}\right)=\langle\bar{u}\rangle_{\mathrm{lo}}\left(z_{h}-D / 2\right)+\frac{d\langle\bar{u}\rangle_{w}}{d \ln z} \times \ln \left(\frac{z_{h}}{z_{h}-D / 2}\right) .
$$

The logarithmic derivative $d\langle\bar{u}\rangle_{w} / d \ln z$ is the slope (in the lin-log plot) of the profile within the wind-turbine region. Its value will be determined next, and it depends critically upon the mixing of momentum in the wind-turbine region. We model its value by assuming an eddy viscosity behavior,

$$
\nu_{T} \frac{d\langle\bar{u}\rangle_{w}}{d z} \approx u_{*}^{2} \rightarrow z \frac{d\langle\bar{u}\rangle_{w}}{d z}=\frac{d\langle\bar{u}\rangle_{w}}{d \ln z}=\frac{z u_{*}^{2}}{\nu_{T}} .
$$

In the region outside the direct influence of the wind turbines, one expects the eddy viscosity to be $\nu_{T}=\kappa u_{*} z$. However, in the wind-turbine region it must be augmented due to the increased turbulence levels there caused by the wakes that are themselves caused by the momentum defect associated with the wind turbines. The momentum defect caused by the wind turbines is proportional to $\frac{1}{2} c_{\mathrm{ft}}\left[\langle\bar{u}\rangle\left(z_{h}\right)\right]^{2}$, from which a turbulence velocity scale may be estimated as $\sqrt{\frac{1}{2} c_{\mathrm{ft}}}\langle\bar{u}\rangle\left(z_{h}\right)$. Using the wake length-scale $D$, a "wake augmented eddy viscosity" can then be estimated as

$$
\nu_{T} \approx \kappa u_{*} z+\sqrt{\frac{c_{\mathrm{ft}}}{2}}\langle\bar{u}\rangle D .
$$

Replacing into Eq. (33) and forming the logarithmic derivative gives

$$
\frac{d\langle\bar{u}\rangle_{w}}{d \ln z}=\frac{u_{*}}{\kappa} \frac{1}{1+\nu_{w}^{*}},
$$

where $\nu_{w}^{*}$ is a nondimensional "wake eddy viscosity" defined as

$$
z_{0, \mathrm{hi}}=z_{h}\left(1+\frac{D}{2 z_{h}}\right)^{\nu_{w}^{*} /\left(1+\nu_{w}^{*}\right)} \exp \left(-\left[\frac{c_{\mathrm{ft}}}{2 \kappa^{2}}+\left(\ln \left[\frac{z_{h}}{z_{0, \mathrm{lo}}}\left(1-\frac{D}{2 z_{h}}\right)^{\nu_{w}^{*}\left(1+\nu_{w}^{*}\right)}\right]\right)^{-2}\right]^{-1 / 2}\right) .
$$

$$
\nu_{w}^{*}=\frac{\sqrt{\frac{1}{2} c_{\mathrm{ft}}}(\bar{u}\rangle D}{\kappa u_{*} z}
$$

and where the value of $z$ is taken to be the value at hub height. At this stage, the condition that the velocity be continuous at $z=z_{h}$ may be written as

$$
\begin{array}{r}
\langle\bar{u}\rangle_{\mathrm{lo}}\left(z_{h}-D / 2\right)+\frac{d\langle\bar{u}\rangle_{w}}{d \ln z} \times \ln \left(\frac{z_{h}}{z_{h}-D / 2}\right) \\
=\langle\bar{u}\rangle_{\mathrm{hi}}\left(z_{h}+D / 2\right)+\frac{d\langle\bar{u}\rangle_{w}}{d \ln z} \times \ln \left(\frac{z_{h}}{z_{h}+D / 2}\right) .
\end{array}
$$

In evaluating $d\langle\bar{u}\rangle_{w} / d \ln z$, we use Eq. (33) but in the left hand side of the equation above we use $u_{* 1_{0}}$ as the appropriate friction velocity (momentum flux) for the lower half of the wind-turbine region, while $u_{* \mathrm{hi}}^{2}$ is used for the upper half in evaluating the right hand side of the above equation. The use of the hi and lo friction velocities (instead of perhaps more accurate intermediate values) is dictated by the desire to maintain simplicity in the resulting expressions (see below). Also, the eddy-viscosity assumption is highly approximate anyhow. The relationship between friction velocities can then be written as

$$
u_{* \mathrm{lo}}=u_{* \mathrm{hi}} \frac{\ln \left[\frac{z_{h}}{z_{0, \mathrm{hi}}}\left(1+\frac{D}{2 z_{h}}\right)^{\nu_{w}^{*} /\left(1+\nu_{w}^{*}\right)}\right]}{\ln \left[\frac{z_{h}}{z_{0, \mathrm{lo}}}\left(1-\frac{D}{2 z_{h}}\right)^{\nu_{w}^{*} /\left(1+\nu_{w}^{*}\right)}\right]} .
$$

Replacing this into the momentum balance and solving for $z_{0, \text { hi }}$ yields
In the limit $\nu_{w}^{*}=0$, one recovers the Frandsen model since the additional mixing in the wake is neglected. This is appropriate for very sparsely distributed or weakly loaded windturbine arrays. The other limit, $\nu_{w}^{*} \rightarrow \infty$, is equivalent to a perfectly mixed, constant mean velocity layer across the wind-turbine region.

To complete the model, one needs to prescribe $\nu_{w}^{*}$. To obtain a numerical value that maintains explicit dependence on $c_{\mathrm{ft}}$, we choose $D \approx z_{h}$ and use $\langle\bar{u}\rangle / u_{*} \approx \kappa^{-1} \ln \left(z_{h} / z_{0}\right)$. Taking a representative value $z_{h} \sim 100 \mathrm{~m}$ and $z_{0} \sim 1 \mathrm{~m}$ (of course, this could be improved through subsequent iterations 


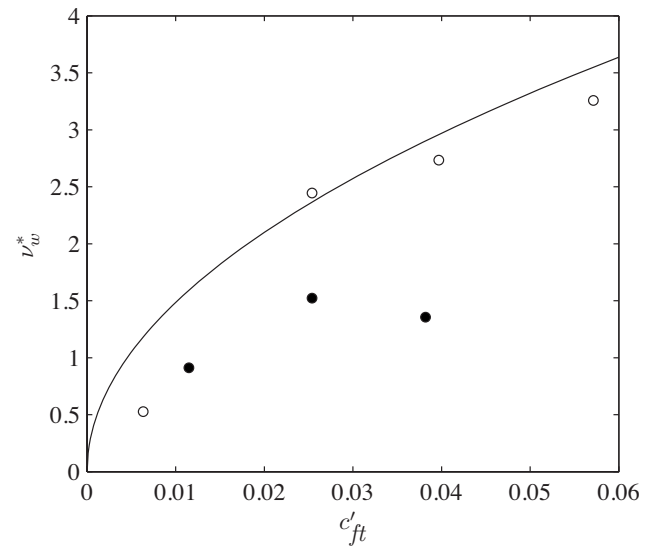

FIG. 10. Wake eddy viscosity $\nu_{w}^{*}$ obtained from LES $(\bigcirc)$ using the measured mean velocity gradient at hub height, compared to the model of Eq. (40) (一), for different $c_{\mathrm{ft}}^{\prime}$ values-( $\left.\bigcirc\right)$ : variation in $c_{\mathrm{ft}}^{\prime}$ by varying the geometric loading $4 s_{x} s_{y} / \pi$ (cases $\mathrm{G}, \mathrm{A} 3, \mathrm{H}$, and I); $(\bullet)$ : variation in $c_{\mathrm{ft}}^{\prime}$ by varying $C_{T}^{\prime}$ (cases $\mathrm{C}, \mathrm{A} 2$, and $\mathrm{B}$ ).

$$
\nu_{w}^{*}=\frac{u_{*, \mathrm{hi}}}{\kappa z_{h} d\langle\bar{u}\rangle /\left.d z\right|_{z=z_{h}}}-1
$$

The mean velocity gradient at hub height is obtained from the LES mean velocity profile using finite difference across the corresponding grid points at $z_{h}$. This "measured" wake viscosity from the LES is compared in Fig. 10 to the simple model of Eq. (40). As may be appreciated, the model provides a reasonable level of agreement with the wake viscosity obtained from LES. Some discrepancies may be observed, in particular, for case B (with $C_{T}^{\prime}=2$, and $c_{\mathrm{ft}}^{\prime}=0.038$ ). This may be related to the evaluation of the mean-velocity derivative, a property that tends to be susceptible to numerical and modeling errors, but apart from that, this also illustrates the accuracy limitations one might expect from the rather crude estimation used for $\nu_{w}^{*}$. Nevertheless, we find the proposed estimation of $\nu_{w}^{*}$ of sufficient accuracy for the purpose of developing the simplified roughness model, as further addressed next.

Finally, the effective roughness length scale may now be obtained from Eqs. (39) and (40). The results are presented in Fig. 9. As can be seen, the agreement with the LES data of the new model is significantly improved over the prior models. The comparison is presented in a different form in Figs. 11(a) and 11(b). In Fig. 11(a), the effective roughness is presented as a function of $c_{\mathrm{ft}}^{\prime}$ for fixed $z_{0,10}$. As can be seen, the agreement of the new model with the LES data is quite good. Moreover, Fig. 11(b) shows the values as a function of $z_{0,10}$ for a fixed value of $c_{\mathrm{ft}}^{\prime}$. The agreement of the new model with the LES data is reasonable, but should be further improved.

Figure 12 shows a comparison between bottom friction velocities obtained from simple models $\left(u_{* \mathrm{l}, m}\right)$ and those arising from LES $\left(u_{* 1 \mathrm{o}, \mathrm{LES}}\right)$ shown in Table II. The comparison includes the original Frandsen formula (stars) and the proposed modified Frandsen formula (open circles). The Lettau approach does not provide a prediction for this friction velocity. We conclude, in terms of predicting the bottom
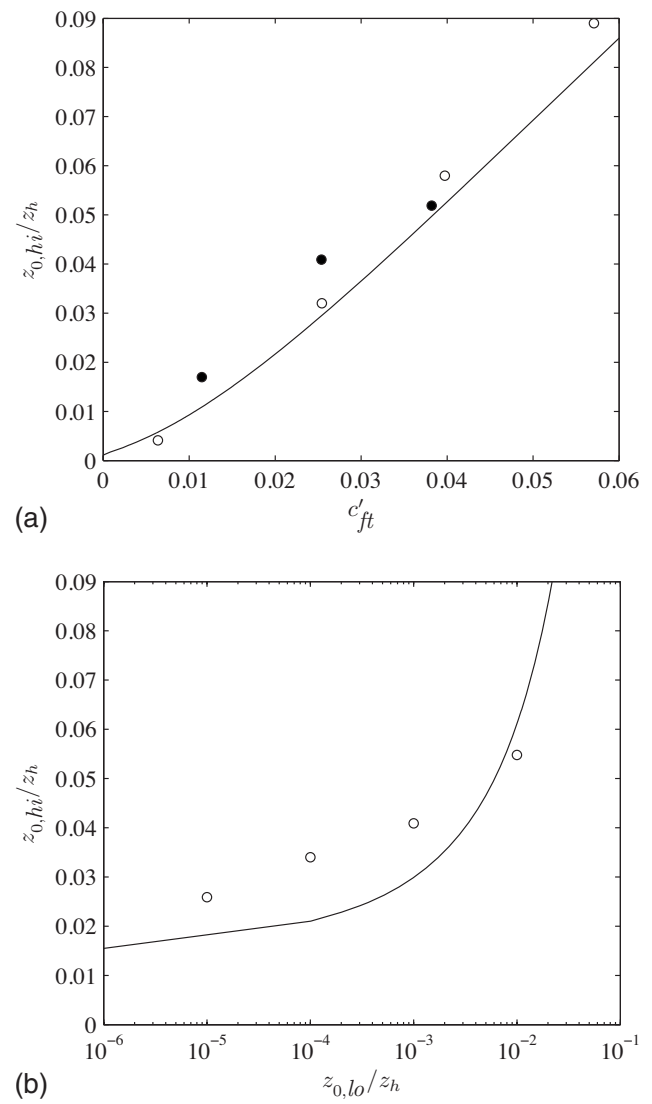

FIG. 11. Evaluation of effective roughness height $z_{0, \mathrm{hi}}$ as a function of $c_{\mathrm{ft}}^{\prime}$ (with $z_{0, \mathrm{l}_{\mathrm{o}}}=10^{-4}$ ) (a) and as a function of $z_{0, \mathrm{lo}}$ (with $c_{\mathrm{ft}}^{\prime}=0.025$ ) (b). Lines correspond to the modified formula for $z_{0, \mathrm{hi}}$. Symbols correspond to LES evaluations. In (a) - $(\bigcirc)$ : variation in $c_{\mathrm{ft}}^{\prime}$ by varying the geometric loading $4 s_{x} s_{y} / \pi$ (cases G, A3, H, and I); $(\bullet)$ : variation in $c_{\mathrm{ft}}^{\prime}$ by varying $C_{T}^{\prime}$ (cases C, $\mathrm{A} 2$, and $\mathrm{B})$.

stress, that both the original Frandsen formula and the modified model provide a good estimate of the bottom friction velocity. These values are of great importance in quantifying mean surface transport properties below wind-turbine arrays.

\section{CONCLUSIONS}

The present simulation study has focused on the "fully developed wind-turbine array boundary layer." This concept becomes relevant for wind farms whose length exceeds the height of the ABL by over an order of magnitude. In this asymptotic regime, changes in the streamwise direction can be neglected and the relevant exchanges occur in the vertical direction. A suite of LES in which wind turbines are modeled using the classic drag disk concept have been performed in order to quantify the vertical transport of momentum and kinetic energy across the boundary layer. The focus of the present paper is on horizontally averaged statistics. The impact of vertical transport of kinetic energy due to turbulence and mean flow correlations is quantified. It has been shown that the vertical fluxes of kinetic energy are of the same order of magnitude as the power extracted by the wind turbines. Unlike the case of isolated or limited number of wind turbines, in a large array ultimately the kinetic energy must be extracted via vertical fluxes from the faster moving flow above the wind-turbine region. 


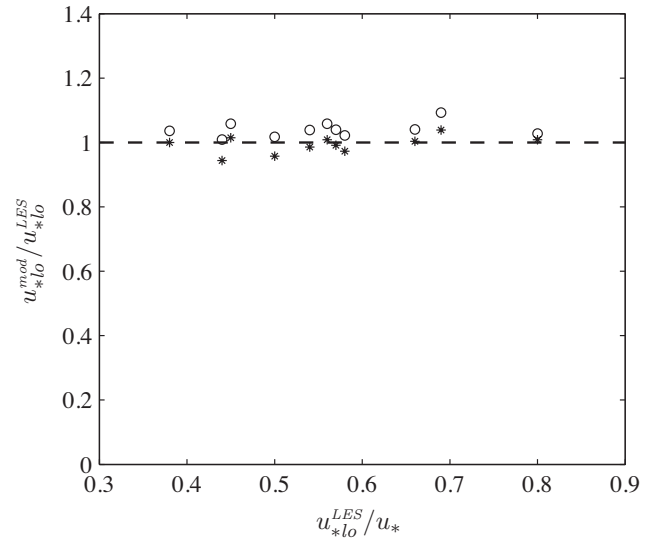

FIG. 12. Comparison between bottom friction velocities obtained from simple models $\left(u_{* 1 \mathrm{lo}}^{\bmod }\right)$ and those arising from LES $\left(u_{* \text { lo }}^{\mathrm{LES}}\right)$. Stars: the original Frandsen formula. Open circles: the proposed modified Frandsen formula.

Present results have been also compared with models for effective roughness length scales. The fundamental assumption of the Frandsen (1992) approach, namely, the existence of two log laws above and below the wind-turbine region, has been verified in the LES results. Based on the observed trends in mean velocity across the wind-turbine region, a modified model is proposed showing improvement in the predicted effective roughness height.

It is useful to remark that the LES results and the proposed modified Frandsen formula for $z_{0 \text {,hi }}$ yield roughness heights that are larger than values predicted by the original Frandsen formula, or those often employed in practice for wind farm modeling. For instance, Ref. 23 used $z_{0, \text { hi }}=0.8 \mathrm{~m}$, i.e., $0.8 \%$ of hub height, for $z_{h}=100 \mathrm{~m}$. For the range of realistic $C_{T}$ and wind-turbine spacings considered in our LES, the obtained values were centered (on a log scale) around $2 \%-3 \%$ of $z_{h}$, i.e., 2-3 $\mathrm{m}$ for a $100 \mathrm{~m}$ height wind turbine, i.e., quite a bit larger than $0.8 \mathrm{~m}$. Of course, when predicting wind reduction factors with geostrophic forcing (see the Appendix), the sensitivity of predicted hub-height velocity is relatively weak stemming from the logarithmic dependence and from the ABL height adjustments that occurs with the increased turbulence levels. However, when cubing the velocity to evaluate effects on power, the effect of the increased $z_{0, \text { hi }}$ can be significant. Thus, for the strongly loaded cases with smaller spacings we believe that the proposed modification to the Frandsen formula is important. The associated insights may also lead to a better understanding of the commonly observed "wind-farm underperformance," which is often attributed to poorly understood wake effect in an ABL environment.

The result of this study was obtained using LES with a drag disk model for the effects of wind turbines. Naturally, more detailed modeling including moving blades etc. would be more accurate, but then covering the entire boundary layer with multiple wind turbines would not be practical, especially when one wishes to compute a suite of different cases with varying parameters, etc. We point out that ongoing experimental measurements in a wind tunnel, in which model wind turbines are used that include moving blades, etc., confirm the general trends for the horizontally averaged profiles of mean velocity and Reynolds stresses observed in the present simulations (although they differ in some details since the experiments are not in the fully developed regime). Overall, we believe that the "drag disk" approach captures the main aspects of the wind-turbine boundary layer interactions. Another approximation used here is the pressuregradient forcing. As discussed in the Appendix, the present results can be used in the context of geostrophic wind forcing as well. Future studies will focus on the spatial structure of the individual wakes within the WTABL, as well as on additional effects from thermal stratification.

\section{ACKNOWLEDGMENTS}

M.C. was supported by (Swiss) SNF 200021-107910/1 land-atmosphere interaction over complex terrain: large eddy simulation and field experiments. He also thanks the encouragement, advice, and support of his main advisor Professor Marc B. Parlange at EPFL. C.M. acknowledges partial funding from the National Science Foundation's Energy for Sustainability Program (Project No. CBET 0730922). J.M. acknowledges funding from the Research Foundation-Flanders (FWO-Vlaanderen).

\section{APPENDIX: IMPLICATIONS OF PRESSURE GRADIENT VERSUS GEOSTROPHIC WIND FORCING}

Consider a classic boundary layer below the geostrophic wind, in which the surface layer direction is such that the flow there is in the $x$-direction. Following Tennekes and Lumley $^{47}$ (p. 169), in the streamwise direction the Reynolds equation and classic similarity analysis lead to the innerlayer solution

$$
\frac{\bar{u}(z)}{u_{*}}=\frac{1}{\kappa} \ln \left(\frac{z}{z_{0}}\right)
$$

and the outer layer solution

$$
\frac{\bar{u}(z)-U_{G}}{u_{*}}=\frac{1}{\kappa} \ln \left(\frac{z f}{u_{*}}\right)+C,
$$

where

$$
f=2 \Omega \sin \phi
$$

is the Coriolis parameter. For $\Omega=2 \pi /(24 \times 3600 \mathrm{~s})=7.27$ $\times 10^{-5} 1 / \mathrm{s}$, and, e.g., at 40 degree latitude, we get $f=9.34$ $\times 10^{-5} 1 / \mathrm{s}$. In the matching range, both velocities $\bar{u}(z)$ are equal and this yields the relation between $U_{G}$ and $u_{*}$,

$$
\frac{U_{G}}{u_{*}}=\frac{1}{\kappa} \ln \left(\frac{u_{*}}{f z_{0}}\right)-C .
$$

The constant $C \approx 4$ according to Tennekes and Lumley. ${ }^{47}$ Frandsen et al. ${ }^{33}$ quoted a value of $A=1.8$, which is related to $C$ according to $A / \kappa=C$ and gives $C=4.5$. This is an implicit equation for $u_{*}$. Following the suggestion used in Ref. 33 for the full geostrophic velocity, but here only for the $U_{G}$ component, a useful (implicit) approximation of Eq. (A4) may be proposed 


$$
U_{G}=\frac{u_{*}}{\kappa}\left[\ln \left(\frac{U_{G}}{f z_{0}}\right)-C_{*}\right],
$$

and we find that this works well for $C_{*}=4.5$. From this, we can now solve for $u_{*}$ and obtain

$$
u_{*}=\frac{\kappa U_{G}}{\left[\ln \left(\frac{U_{G}}{f z_{0}}\right)-C_{*}\right]} .
$$

Thus if we wish to match the velocity far above the windturbine array to a given $U_{G}$, this expression allows us to determine the corresponding $u_{*}$ for any given $z_{0}$. In order to find at what height, $z=H_{G}$, the mean velocity merges with $U_{G}$, we use

$$
0=\frac{1}{\kappa} \ln \left(\frac{H_{G} f}{u_{*}}\right)+C
$$

and obtain

$$
H_{G}=\exp (-\kappa C) \frac{u_{*}}{f} \approx 0.16 \frac{u_{*}}{f} .
$$

For typical dimensional values of $u_{*}$ (say, $u_{*}=0.45 \mathrm{~m} / \mathrm{s}$ ) and $f=9.34 \times 10^{-5} 1 / \mathrm{s}$, this yields $H_{G}=770 \mathrm{~m}$, comparable to the domain size usually assumed. The corresponding value of $U_{G} / u_{*}$ obtained from Eq. (A4) is $U_{G} / u_{*}=22.5$, which is $U_{G}=10 \mathrm{~m} / \mathrm{s}$.

For the case with many wind turbines, we obtained from LES values in a range $z_{0, \mathrm{hi}} / H$ ranging from $0.41 \times 10^{-3}$ to $8.9 \times 10^{-3}$. For the sake of discussion, let us discuss a case with $z_{0, \mathrm{hi}}=2.6 \mathrm{~m}$ (case F). Then for the same value of $U_{G}$, Eq. (A6) gives $u_{*}=0.66 \mathrm{~m} / \mathrm{s}$. Compared to $0.45 \mathrm{~m} / \mathrm{s}$, this represents an increase of a factor of 1.47 for the friction velocity and 2.15 for the stresses and turbulence variances. The corresponding height of the new boundary layer height is $H_{G}^{\prime}=0.16 \times 0.66 / f=1130.6 \mathrm{~m}$. The simulation domain is still quite adequate for this. Note that the top velocity value in the simulation for the baseline case is $\bar{u}(z / H=1) / u_{*}$ $\approx 15.0$. With $u_{*}=0.66 \mathrm{~m} / \mathrm{s}$, this still means a streamwise geostrophic velocity of $10 \mathrm{~m} / \mathrm{s}$, as required by the consistency conditions.

Often, the full geostrophic wind velocity $\mathcal{G}=\sqrt{U_{G}^{2}+V_{G}^{2}}$ is prescribed. For the $V_{G}$ component, the classical derivation yields ${ }^{47} V_{G} / u_{*}=-A$, with $A \sim 12$. Frandsen et al. ${ }^{33}$ quoted a value of $B=4.5$, which is similar to the Tennekes and Lumley ${ }^{47}$ value with $A=B / \kappa=4.5 / 0.4=11.25$. As a result, for the no-wind-turbine case of $u_{*}=0.45 \mathrm{~m} / \mathrm{s}$, we get $V_{G}=-5 \mathrm{~m} / \mathrm{s}$, i.e., the bottom flow near the ground is tilted by $\tan ^{-1}(5 / 10)=26^{\circ}$ with respect to the geostrophic wind. In the simulation, we disregard the transverse mean velocity that would be needed to merge with the geostropic wind there. It is expected to have very little effect in the nearground region. For the case with wind turbines, we get $V_{G}$ $=-11.25 \times 0.66=-7.43 \mathrm{~m} / \mathrm{s}$. That is to say, the near-ground flow is shifted some more, by $\tan ^{-1}(7.43 / 10)=36.6^{\circ}$ with respect to the geostrophic wind.

In terms of reduction in resource, the velocity at the wind-turbine location for case F (see Fig. 7) is about $\langle\bar{u}\rangle / u_{*} \sim 9.1$. In the case of a constant geostrophic velocity forcing described in the preceding paragraph $\left(U_{G}=10 \mathrm{~m} / \mathrm{s}\right)$, and a friction velocity of $u_{*}=0.66 \mathrm{~m} / \mathrm{s}$, the turbine-height predicted velocity is therefore about $6 \mathrm{~m} / \mathrm{s}$. This has to be compared with the "unperturbed" case without wind-turbine arrays with $U_{G}=10 \mathrm{~m} / \mathrm{s}$ for which the mean velocity at turbine height is $\langle\bar{u}\rangle / u_{*}=\kappa^{-1} \ln (100 / 0.1) \sim 17.3$. With $u_{*}=0.45 \mathrm{~m} / \mathrm{s}$, this gives $7.8 \mathrm{~m} / \mathrm{s}$. Therefore, the velocity reduction factor for the baseline case is about 6/7.8 0.77. Raised to the third power, this gives a factor of 0.46 . Thus, for the arrangement of the baseline case, the power extracted from the infinite array is significantly reduced compared to the case of a single turbine.

In regard to LES using $x_{1}$-direction pressure forcing as done in the present paper, the standard approach using LES with Coriolis effects and geostrophic velocity forcing is to include a term $+f\left(\widetilde{u}_{2}-V_{G}\right)$ in the streamwise direction momentum equation's right-hand side (instead of an applied pressure gradient), and a term $-f\left(\widetilde{u}_{1}-U_{G}\right)$ in the transverse direction equation. The former (streamwise direction) includes the Coriolis acceleration proportional to $-2 \Omega_{3} \widetilde{u}_{2}$ and the pressure gradient part that is in geostrophic balance: $\rho^{-1} \partial p_{G} / \partial x_{1}=2 \Omega_{3} V_{G}$. Thus, in our simulation we neglect $-2 \Omega_{3} \tilde{u}_{2}$ (the Coriolis acceleration due to the transverse velocity, which has zero mean anyhow). Also, in the transverse direction, not having a pressure gradient is equivalent to neglecting the fluctuations of $\widetilde{u}_{1}$ away from $U_{G}$.

Imposing a streamwise pressure gradient in the LES $x_{1}$-direction is thus equivalent to assuming a given value for the transverse geostrophic wind according to $\rho^{-1} \partial p_{G} / \partial x_{1}$ $=2 \Omega_{3} V_{G}=f V_{G}$. Replacing $f$ in terms of the traditional scaling between $f, H_{G}$, and $u_{*}$ described above, this is therefore equivalent to imposing $\rho^{-1} \partial p_{G} / \partial x_{1}=\exp (-\kappa C) u_{*} / H_{G} \times V_{G}$ $=-A \exp (-\kappa C) u_{*}^{2} / H_{G}$. The standard numerical values $A=11.25$ and $C=4.5$ yield $\rho^{-1} \partial p_{G} / \partial x_{1}=-1.8 u_{*}^{2} / H_{G}$. This is of the same order of magnitude as the usual relationship between applied pressure forcing in the streamwise direction in a computational domain size $H$, i.e., $\rho^{-1} \partial p / \partial x_{1}=-u_{*}^{2} / H$. The LES results from the approach of using only an applied $x_{1}$-direction pressure gradient are thus quite relevant to the case with geostrophic forcing, as long as the "turning" effects can be neglected between the ground and the region including the wind-turbine height and slightly above. There are expected to be growing levels of differences with the mean flow direction above the surface layer region of course, and also for the large-scale structures overhead that may be more susceptible to Coriolis accelerations. For the purposes of the present paper, however, the pressure gradient forcing yields useful results.

${ }^{1} \mathrm{H}$. Snel, "Review of the present status of rotor aerodynamics," Wind Energy 1, 46 (1998).

${ }^{2}$ T. Burton, D. Sharpe, N. Jenkins, and E. Bossanyi, Wind Energy Handbook (Wiley, New York, 2001).

${ }^{3}$ A. Crespo and J. Hernández, "Turbulence characteristics in wind-turbine wakes," J. Wind Eng. Ind. Aerodyn. 61, 71 (1996).

${ }^{4}$ J. Whale, C. G. Anderson, R. Bareiss, and S. Wagner, "An experimental and numerical study of the vortex strcture in the wake of a wind turbine," J. Wind Eng. Ind. Aerodyn. 84, 1 (2000).

${ }^{5}$ L. A. Ivanova and E. D. Nadyozhina, "Wind flow deformation inside the wind farm," J. Wind Eng. Ind. Aerodyn. 74-76, 389 (1998). 
${ }^{6}$ P. R. Ebert and D. H. Wood, "The near wake of a model horizontal-axis wind turbine-I. Experimental arrangements and initial results," Renewable Energy 12, 225 (1997).

${ }^{7}$ P. R. Ebert and D. H. Wood, "The near wake of a model horizontal-axis wind turbine-II. General features of the three-dimensional flow field," Renewable Energy 18, 513 (1999).

${ }^{8}$ M. Magnusson and A.-S. Smedman, "Air flow behind wind turbine," J. Wind Eng. Ind. Aerodyn. 80, 169 (1999).

${ }^{9}$ L. J. Vermeer, J. N. Sorensen, and A. Crespo, "Wind turbine wake aerodynamics," Prog. Aerosp. Sci. 39, 467 (2003).

${ }^{10}$ J. N. Sorensen and W. Z. Shen, "Numerical modeling of wind turbine wakes," J. Fluids Eng. 124, 393 (2002).

${ }^{11}$ D. Medici and P. H. Alfredsson, "Measurement on a wind turbine wake: 3d effects and bluff body vortex shedding," Wind Energy 9, 219 (2006).

${ }^{12}$ L. Chamorro and F. Porte-Agel, "A wind-tunnel investigation of windturbine wakes: Boundary-layer turbulence effects," Boundary-Layer Meteorol. 132, 129 (2009).

${ }^{13}$ U. Hogstrom, D. N. Asimakopoulos, H. Kambezidis, C. G. Helmis, and A. Smedman, "A field study of the wake behind a 2 MW wind turbine," Atmos. Environ. 22, 803 (1988).

${ }^{14}$ J. Kline, "Turbulence characteristics at Howden Wind Park I," AWEA Conference "Windpower," Honolulu, HI, 1988.

${ }^{15}$ J. Van Leuven and D. Stevens, "The wind farm of Zeebrugge: Experimental set-up," J. Wind Eng. Ind. Aerodyn. 27, 139 (1988).

${ }^{16}$ S. G. Voutsinas, K. G. Rados, and A. Zervos, "On the analysis of wake effects in wind parks," J. Wind Eng. Ind. Aerodyn. 14, 204 (1990).

${ }^{17} \mathrm{D}$. Smith, "Multiple wake measurements and analysis," in Proceedings of the 12th BWEA Wind Energy Conference, Norwich, UK, edited by T. D. Davies, J. A. Halliday, and J. P. Palutikov (British Wind Energy Association, London, 1990), pp. 53-56.

${ }^{18}$ U. Hassan, A. G. Glendinning, and C. A. Morgan, "A wind tunnel investigation of the wake structure and machine loads within small wind turbine farms," in Proceedings of the 12th BWEA Wind Energy Conference, Norwich, UK, edited by T. D. Davies, J. A. Halliday, and J. P. Palutikov (British Wind Energy Association, London, 1990), pp. 47-52.

${ }^{19} \mathrm{~S}$. Frandsen, "On the wind speed reduction in the center of large clusters of wind turbines," J. Wind Eng. Ind. Aerodyn. 39, 251 (1992).

${ }^{20}$ P. B. S. Lissaman, "Energy effectiveness of arbitrary arrays of wind turbines," Proceedings of the 17th AIAA Aerospace Sciences Meeting, New Orleans, LA, January, 1979, Paper 79-0114.

${ }^{21}$ D. Keith, J. DeCarolis, D. Denkenberger, D. Lenschow, S. Malyshev, S. Pacala, and P. J. Rasch, "The influence of large-scale wind power on global climate," Proc. Natl. Acad. Sci. U.S.A. 101, 16115 (2004).

${ }^{22}$ S. Baidya-Roy, S. W. Pacala, and R. L. Walko, "Can large scale wind farms affect local meteorology?," J. Geophys. Res. 109, D19101, doi:10.1029/2004JD004763 (2004).

${ }^{23}$ D. Barrie and D. Kirk-Davidoff, "Weather response to management of large wind turbine array," Atmos. Chem. Phys. Discuss. 9, 2917 (2009).

${ }^{24}$ J. Jimenez and R. D. Moser, "What are we learning from simulating wall turbulence?," Philos. Trans. R. Soc. London 365, 715 (2007).

${ }^{25}$ K. R. Sreenivasan, "The turbulent boundary layer," Frontiers in Experimental Fluid Mechanics (Springer-Verlag, Berlin, 1989), pp. 159-209.

${ }^{26}$ M. R. Raupach, R. A. Antonia, and S. Rajagopalan, "Rough-wall turbulent boundary layers," Appl. Mech. Rev. 44, 1 (1991).

${ }^{27}$ J Finnigan, "Turbulence in plant canopies," Annu. Rev. Fluid Mech. 32, 519 (2000)
${ }^{28}$ J. Kutzbach, "Investigations of the modification of wind profiles by artificially controlled surface roughness," Studies of the Three Dimensional Structure of the Planetary Boundary Layer (Annual Rept. Department of Meteorology, University of Wisconsin, Madison, 1961), pp. 71-113.

${ }^{29} \mathrm{H}$. Lettau, "Note on aerodynamic roughness-parameter estimation on the basis of roughness-element description," J. Appl. Meteor. 8, 828 (1969).

${ }^{30}$ R. J. Templin, "An estimation of the interaction of windmills in widespread arrays," Laboratory Report No. LTR-LA-171 (National Aeronautical Establishment, Otawa, 1974).

${ }^{31}$ B. J. Newman, "The spacing of wind turbines in large arrays," Energy Convers. 16, 169 (1977).

${ }^{32}$ E. A. Bossanyi, C. Maclean, G. E. Whittle, P. D. Dunn, N. H. Lipman, and P. J. Musgrove, "The efficiency of wind turbine clusters," Proceedings of the Third International Symposium on Wind Energy Systems (BHRA), 1980, pp. 401-416.

${ }^{33}$ S. Frandsen, R. Barthelmie, S. Pryor, O. Rathmann, S. Larsen, J. Hojstrup, and M. Thogersen, "Analytical modelling of wind speed decit in large offshore wind farms," Wind Energy 9, 39 (2006).

${ }^{34}$ C.-H. Moeng, "A large-eddy simulation model for the study of planetary boundary-layer turbulence," J. Atmos. Sci. 6, 2311 (1984).

${ }^{35}$ J. D. Albertson and M. B. Parlange, "Surface length-scales and shear stress: Implications for land-atmosphere interaction over complex terrain," Water Resour. Res. 35, 2121, doi:10.1029/1999WR900094 (1999).

${ }^{36}$ M. Germano, U. Piomelli, P. Moin, and W. H. Cabot, "A dynamic subgridscale eddy viscosity model," Phys. Fluids A 3, 1760 (1991).

${ }^{37}$ E. Bou-Zeid, C. Meneveau, and M. B. Parlange, "A scale-dependent Lagrangian dynamic model for large eddy simulation of complex turbulent flows," Phys. Fluids 17, 025105 (2005).

${ }^{38}$ R. W. C. P. Verstappen and A. E. P. Veldman, "Symmetry-preserving discretization of turbulent flow," J. Comput. Phys. 187, 343 (2003).

${ }^{39}$ J. Smagorinsky, "General circulation experiments with the primitive equations. I. The basic experiment," Mon. Weather Rev. 91, 99 (1963).

${ }^{40}$ P. J. Mason and D. J. Thomson, "Stochastic backscatter in large-eddy simulations of boundary layers," J. Fluid Mech. 242, 51 (1992).

${ }^{41}$ C. Canuto, M. Y. Hussaini, A. Quarteroni, and T. A. Zang, Spectral Methods in Fluid Dynamics (Springer-Verlag, Berlin, 1988).

${ }^{42}$ M. Frigo and S. G. Johnson, "The design and implementation of FFTW3," Proc. IEEE 93, 216 (2005), special issue on program generation, optimization, and platform adaptation.

${ }^{43}$ A. Jimenez, A. Crespo, E. Migoya, and J. Garcia, "Advances in large-eddy simulation of a wind turbine wake," J. Phys.: Conf. Ser. 75, 012041 (2007).

${ }^{44}$ A. Jimenez, A. Crespo, E. Migoya, and J. Garcia, "Large-eddy simulation of spectral coherence in a wind turbine wake," Environ. Res. 3, 015004 (2008).

${ }^{45}$ J. Meyers and C. Meneveau, "Large eddy simulations of large windturbine arrays in the atmospheric boundary layer," AIAA Paper No. 2010$827,2010$.

${ }^{46}$ R. B. Cal, J. Lebrón, H. S. Kang, L. Castillo, and C. Meneveau, "Experimental study of the horizontally averaged flow structure in a model windturbine array boundary layer," J. Renewable Sustainable Energy (to be published).

${ }^{47}$ H. Tennekes and J. L. Lumley, A First Course in Turbulence (MIT Press, Cambridge, MA, 1972). 